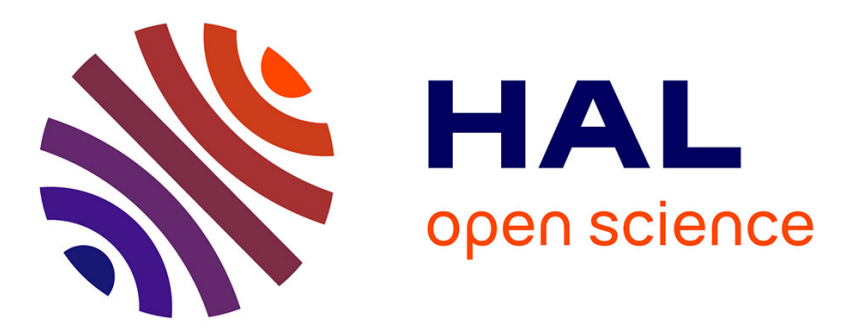

\title{
Evaluation of temperature field and heat flux by inverse analysis during steel strip rolling
}

Daniel Weisz-Patrault, Alain Ehrlacher, Nicolas Legrand

\section{To cite this version:}

Daniel Weisz-Patrault, Alain Ehrlacher, Nicolas Legrand. Evaluation of temperature field and heat flux by inverse analysis during steel strip rolling. International Journal of Heat and Mass Transfer, 2012, 55 (4), pp.629-641. 10.1016/j.ijheatmasstransfer.2011.10.048 . hal-00649450

\section{HAL Id: hal-00649450 \\ https://hal-enpc.archives-ouvertes.fr/hal-00649450}

Submitted on 8 Dec 2011

HAL is a multi-disciplinary open access archive for the deposit and dissemination of scientific research documents, whether they are published or not. The documents may come from teaching and research institutions in France or abroad, or from public or private research centers.
L'archive ouverte pluridisciplinaire HAL, est destinée au dépôt et à la diffusion de documents scientifiques de niveau recherche, publiés ou non, émanant des établissements d'enseignement et de recherche français ou étrangers, des laboratoires publics ou privés. 


\title{
Evaluation of temperature field and heat flux by inverse analysis during steel strip rolling
}

\author{
Daniel Weisz-Patrault ${ }^{\mathrm{a}}$, Alain Ehrlacher ${ }^{\mathrm{a}}$, Nicolas Legrand ${ }^{\mathrm{b}}$ \\ ${ }^{a}$ Ecole Ponts ParisTech, UR Navier, 6 \& 8 Ave Blaise Pascal, 77455 Marne La Vallee, France \\ ${ }^{b}$ ArcelorMittal Global Research \& Development, Maizière Process Voie Romaine, BP 30320 F-57283 Maizières-lès-Metz Cedex
}

\begin{abstract}
Knowledge of the temperature field in the roll is a critical factor of modern, high-speed rolling mills. In this paper, an inverse analytical method is developed to determine the temperature field and especially the temperature (and heat flux) at the surface of the roll by measuring the temperature with a thermocouple (fully embedded) at only one point inside the roll. Iterative methods are not studied because short computation times are desired. Some assumptions are done to resolve analytically the unsteady heat equation, taking into account the restrictions of the measurement system (e.g., measurement according to successive times). The solution is validated by comparing the outputs of the method and prescribed analytical temperature fields. Good agreement is obtained. Noise sensitivity is estimated by adding artificial random numbers to the inputs. Good accuracy is observed. A $10 \%$ error of the temperature sensor depth is also considered and does not compromise the method. On the other hand, the computation time (around 0.05 second by cycle) is studied to rapidly optimise the industrial parameters during the rolling process.
\end{abstract}

Keywords:

Steel rolling, Thermal sensor, Free from damage, Computation time, Inverse analysis

\begin{tabular}{|ll|}
\hline$R_{s}$ & Outer radius (radius of the surface of the roll) \\
$R_{m}$ & Inner radius (radius of the measurements) \\
$e$ & Error of the sensor depth \\
$\omega$ & Rotation speed \\
$r$ & Radial position \\
$\varphi$ & Angular position \\
$t$ & Time \\
$k$ & Index of the current cycle \\
$t_{k}$ & Time at the beginning of the $k$ th cycle $(=2(k-1) \pi / \omega)$ \\
$t_{\varphi}^{k}$ & Time related to the angular position $\left(=t_{k}+\varphi / \omega\right)$ \\
$f$ & Frequency of acquisition of the measurements \\
$\lambda$ & Thermal conductivity of the roll \\
$D$ & Thermal diffusivity of the roll \\
$\epsilon$ & Percentage of error \\
$T$ & Temperature field (solution) \\
$F_{n}$ & $n$th coefficient of Fourier of $T$ \\
$T_{1}$ & First part of the temperature field (solution) \\
$T_{2}$ & Second part of the temperature field (solution) \\
$T^{m}$ & Measured temperatures (inputs) \\
$\widehat{T}_{n}^{k}$ & $n$th coefficient of Fourier of $T^{m}$ (kth cycle) \\
$T^{s}$ & Temperature at the surface of the roll (outputs) \\
$T_{k}^{s}$ & Vector of the computed outputs (cycle $k$ ) \\
$H^{s}$ & Heat flux at the surface of the roll (outputs) \\
$T^{p}$ & Prescribed temperature field (validation of the method)
\end{tabular}




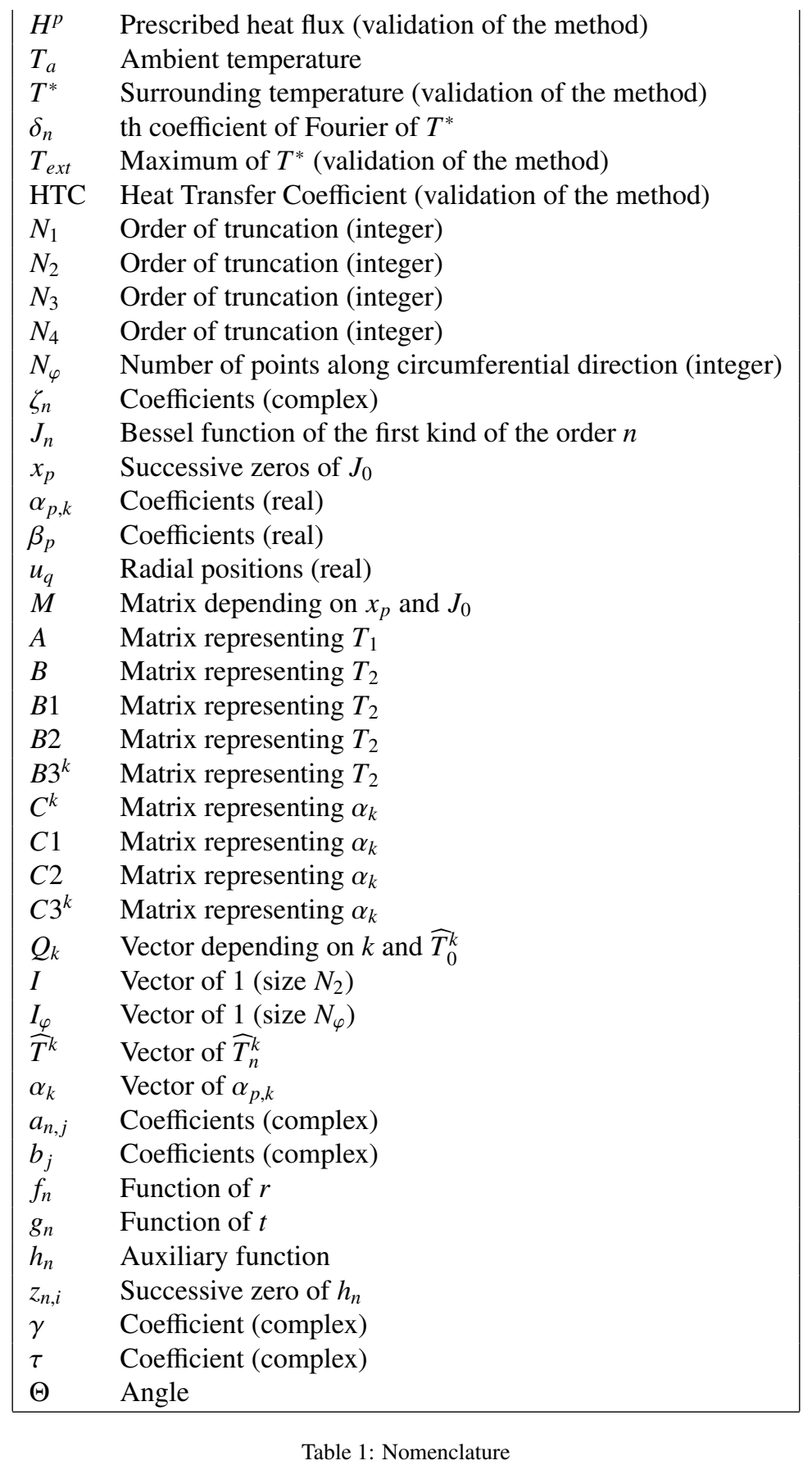

\section{Introduction}

\subsection{Thermal problem}

In steel rolling processes, two rolls are used as tools to reduce the thickness of a workpiece. For hot rolling conditions, knowledge of the heat fluxes entering the roll would allow a better evaluation of the thermal fatigue, which is one of the major factor of wear on rolls, as investigated by Corral et al. [1] who proposed a simplified analytical model for both thermal and thermoelastic problems. Li et al. [2] proposed more recently a threedimensional model by Finite Element Method (FEM) to evaluate the thermal stress of the roll. Fatigue load is completely negligible for cold rolling conditions. However, in the roll gap the characterization of the thermal problem would lead to knowledge of viscosity and thickness of the lubricant. Moreover the roll expansion and thermal crown are important factors of the prediction of the flatness of products. 


\subsection{Predictive models}

Thus many works focus on the thermal problem of rolling mills. Tseng et al. [3] developed a direct analytical steady-state approach. Guo [4] used a semi-analytical method involving Laplace and inverse transforms to pursue the unsteady solution for typical boundary conditions. Tseng [5] proposed a two-dimensional steady-state model based on Finite Difference Method (FDM). Zone-Ching and Chang-Cheng [6] developed a three-dimensional model (by FDM) with complex boundary conditions to predict the temperature field in the roll. In order to optimise the geometry of water spray of the cooling system Saboonchi and Abbaspour [7] proposed a simplified model (by FDM) which computes the three-dimensional temperature field. The equation is computed along the circumferential direction with respect to time (index of time and angular position are the same). Zhang et al. [8], [9] proposed finite difference method or finite element method to predict the temperature field in the roll and thermal crown by neglecting the variations along the circumferential direction but taking into account variations along the axis of the roll. Montmitonnet [10] also proposed a predictive model (by FEM) of the whole rolling process, by coupling the strip and the roll thermal behaviours with iterative methods.

\subsection{Objective of the paper}

All the previous cited works are theoretical. Generally experimental temperature fields are obtained with an infrared camera which evaluates the heat fluxes at the boundary of the body. However intensive lubrication (water/oil emulsion) and cooling make impossible the use of an infrared camera. Therefore an inverse analysis is needed. In this paper, a method to interpret the measurements of a single fully embedded thermocouple is described. The method gives the temperature field in the whole roll. The main outputs of the method are the reconstruction of the temperature at the surface of the roll and the heat fluxes entering the roll, because these are the most useful data. The method developed here is based on an analytical approach of the unsteady heat equation, managing some assumptions in order to take into account the restrictions of the measurement system (e.g., measurement according to successive times). The present method is limited to a two-dimensional description along radial and circumferential directions. If several thermocouples are inserted under the surface of the roll at different positions along the axial direction, then it is possible to obtain a three-dimensional description. However this has not been studied in this paper.

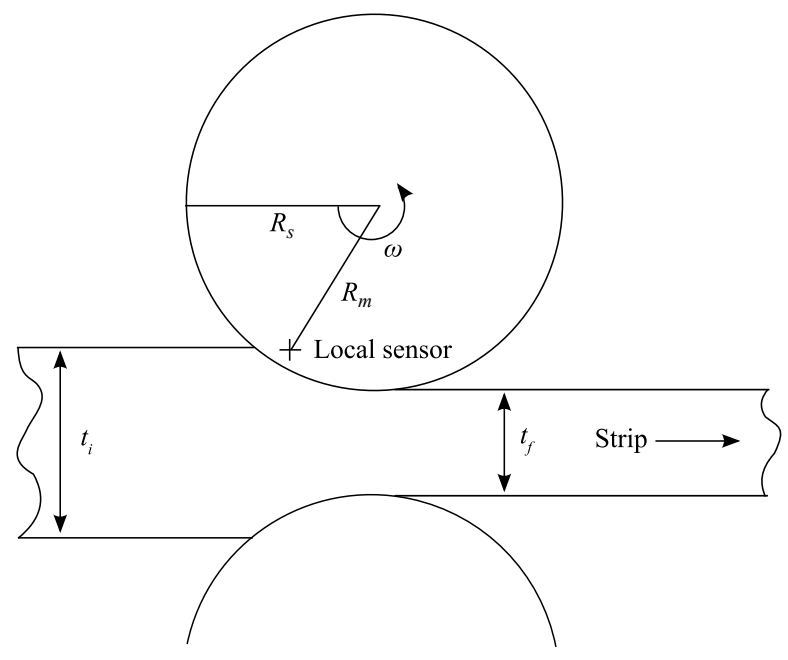

Figure 1: Rolling process

\subsection{Inverse methods and measurement interpretation}

In the general field of thermal inverse analysis, an extensive work has been done. Blum and Marquardt [11] gave an overview of the existing methods and proposed them-self a numerical method based on a low pass filter interpretation of the unsteady heat equation. The most of the presented works are numerical. Raynaud and Bransier [12] with the space marching algorithms, Alifanov [13] with the regularization methods, Beck et al. [14] with the function specification or Scarpa and Milano [15] with Kalman's filter techniques involve all of them a spatial (and/or time) discretization. 
Concerning the specific problem of rolling process several inverse methods already exist. Tseng et al. [16] proposed an inverse FEM. The measurements are used to quantify the heat flux in the roll gap. The heat transfer coefficient in the spray portion is evaluated through an inverse heat conduction (IHC) technique. Many inverse methods are based on matching the inputs or measurements and the outputs of the model equations. Least square method and conjugate gradient method are often used for this purpose. Huang et al. [17] proposed a unsteadystate inverse analysis based on the conjugate gradient method to obtain the heat fluxes in the strip-roll arc of contact by measuring inside the roll body with a fully embedded local sensor. The iterative method (around 35 iterations) consists (for each iteration) in solving numerically the direct problem, the sensitivity problem and the adjoint problem to minimize the error between the estimated temperature and the measured temperature. However, considering the computation time Huang et al. [17] mesh only the first few millimeters of the roll by assuming that the radial heat flux is negligible under a certain depth. For very long durations this assumption is untrue, the temperature profile ranging from the center to the surface of the roll. Hsu et al. [18] and Keanini [19] proposed three-dimensional inverse methods to evaluate heat flux entering the roll by measuring at several different locations. These contributions are based on FDM or FEM, matrix forms and least square method. More recently Chen and Yang [20] proposed an inverse method based on the conjugate gradient technique. The authors mesh the roll completely with a refined mesh near the roll gap. However, a steady state is assumed because the method relies on the steady heat equation.

Several contributions use inverse methods to interpret experimental measurements. Tavares et al. [21] proposed an inverse method based on one-dimensional model (Lagrangian coordinate system moving at the same speed as the roll). The response time of the thermocouple is studied and a proper correction of the temperature data is introduced. Kotrbacek et al. [22] proposed a comprehensive numerical inverse approach, where the thermocouple is modeled to interpret measurements to optimise the cooling of the roll. This paper does not present experimental data and the disturbance (response time etc...) of the thermocouple is not taken into account in the following.

\subsection{Choice of the method}

Some older works propose analytical inverse solutions of the unsteady heat equation such as the ones proposed by Kudryavtsev [23]. For a few special cases these exact methods are quite simple and efficient. In this paper an analytical solution is chosen because by neglecting the heat transfer along the axis of the roll, the two dimensional problem is a circle which is a particularly simple object. Moreover, Weisz-Patrault et al. [24] proposed in a previous work a method to measure the stress in the whole roll by inverse analytical analysis. The problem was supposed to be isothermal. In order to solve the coupled thermo-mechanical problem, the best option is to solve also analytically the thermal problem. Both solutions will be easily overlapped.

On the other hand, the computation time is studied to rapidly optimise the industrial parameters during the rolling process. Numerical method with iterative schemes or very comprehensive approaches which imply long computation times are therefore not studied here. The CPU time (around 0.05 second by cycle) is obtained for a quadcore $2.8 \mathrm{GHz}$ processor and is the time displayed by Scilab 5.3.

\subsection{Validation of the method}

The method takes as inputs the temperature measured under the surface of the roll at the inner radius called $R_{m}(\mathrm{~mm})$ and gives as outputs the temperature (and the heat flux) at the surface of the roll (radius called $R_{S}(\mathrm{~mm})$ ). The depth of the thermocouple is discussed in Section 7 and a $10 \%$ error of the sensor depth is considered in Section 10.4. Usually the unknown boundary condition of an inverse method is obtained by matching the inputs and the outputs of the model equations. In these cases the accuracy of the method is evaluated by analyzing the deviation between measurements and model output to detect bias. The most of the cited works in Section 1.4 use this approach.

The present method does not belong to this class of methods. The successive measured temperatures at the inner radius $R_{m}$ allows a solution everywhere in the roll including the surface of the roll. Therefore the method only consist in finding an analytical solution of the problem bounded by the measurements and extending it by continuity toward the surface of the roll. There is no matching process.

No experimental data were available. Therefore, the inputs (replacing measurements) are extracted from analytical temperature fields (solutions of the unsteady heat equation) close from typical temperature fields that occur during rolling processes as observed with predictive models [1] to [7]. The solution is validated by comparing the outputs of the method and the prescribed analytical temperature at the surface of the roll. The same process is also performed for the heat flux by interpreting the same inputs. The relative difference between the outputs and the prescribed temperature (or heat flux) is used to quantify the quality of the method. Good agreement is obtained. Noise sensitivity is estimated by adding random numbers to the inputs and good accuracy is observed. 


\subsection{Eurlerian reference}

The evolution of the temperature of a material point (Lagrangian description) of the roll is very fast. The time dependence could be difficult to describe, that is why an Eulerian description is developed in order to have a slow evolution of the temperature field (small variations from one cycle to another). Thus, an Eulerian point ( $r$ radial position, $\varphi$ angular position) is not material, that is to say it describes the evolution of a succession of material points at a given position in a fixed reference. In the following, all the quantities are calculated in an Eulerian reference.

\section{Time interval}

If cycles are numbered (index $k$ ) it is convenient to divide the total time interval in subintervals $\left[t_{k}, t_{k+1}\right]$ with $t_{k}=2(k-1) \pi / \omega$ (considering that the rotation speed $\omega(\mathrm{rad} / \mathrm{s})$ is at least constant during each cycle). The inputs are the temperature measured at the radius $R_{m}$. However, since there is only one measurement point, the inputs are given at different angular positions at successive times. The rotation of the roll allows an information on the whole circle (radius $R_{m}$ ) but not at all times. Each angular position is known at one particular time according to the frequency of acquisition $f(\mathrm{~Hz})$ and the rotation speed $\omega$. Therefore the inputs depend only on the angular position $\varphi$ (or corresponding times $\left.t_{\varphi}^{k}=t_{k}+\varphi / \omega\right)$ and on the index of the cycle $k$. Inputs are called $T^{m}(\varphi, k)$ where $m$ means measured (no experiments were available therefore calculated data replace measurements). Notations are listed in Table 1.

\section{Problem}

The solution of the unsteady heat equation is called $T(r, \varphi, t, k)(r$ and $\varphi$ being the radial and angular coordinates, $t$ meaning time and $k$ being the index of the cycle). It should be noted that $t$ and $k$ are related $\left(t \in\left[t_{k}, t_{k+1}\right]\right)$. Therefore the outputs are $T^{s}(\varphi, t, k)=T\left(R_{s}, \varphi, t, k\right)$, where $s$ means surface. The heat flux at the surface of the roll is $H^{s}(\varphi, t, k)=\lambda(\partial T / \partial r)\left(R^{s}, \varphi, t, k\right) . D\left(\mathrm{~m}^{2} / \mathrm{s}\right)$ being the thermal diffusivity of the roll (assumed to be independent on the temperature) and using Eulerian description, the unsteady heat equation governing the temperature field $T(r, \varphi, t, k)$ for a homogeneous and isotropic roll during the $k$ th cycle is:

$$
\frac{\partial^{2} T}{\partial r^{2}}+\frac{1}{r} \frac{\partial T}{\partial r}+\frac{1}{r^{2}} \frac{\partial^{2} T}{\partial \varphi^{2}}=\frac{1}{D}\left(\frac{\partial T}{\partial t}+\omega \frac{\partial T}{\partial \varphi}\right)
$$

With the set of conditions:

$$
\left\{\begin{array}{l}
(r, \varphi, t, k) \in\left[0, R_{s}\right] \times\left[0,2 \pi\left[\times\left[t_{k}, t_{k+1}\right] \times \mathbb{N}^{*}\right.\right. \\
T\left(r, \varphi, t_{k}, k\right)= \begin{cases}T_{a} & k=1 \\
T\left(r, \varphi, t_{k}, k-1\right) & k \geqslant 2\end{cases} \\
T\left(R_{s}, \varphi, t, k\right) \text { (unknown boundary conditions) }
\end{array}\right. \text { (initial condition) }
$$

For the first cycle, the initial condition is the ambient temperature in the entire roll. For the next cycles the initial condition is the temperature field at the end of the previous cycle. Moreover it can be noted that in $r=0$ the radial heat flux should be zero, but this condition is automatically verified (no constant to determine).

In order to solve (1) with the conditions (2), the spatial domain can be split in two parts:

$$
\left\{\begin{array}{l}
\mathcal{D}_{1}=\left[0, R_{m}\right] \times[0,2 \pi[ \\
\left.\left.\mathcal{D}_{2}=\right] R_{m}, R_{s}\right] \times[0,2 \pi[
\end{array}\right.
$$

The principle of the present inverse method is to solve analytically the unsteady heat equation (1) in $\mathcal{D}_{1}$ (i.e., in the sub-domain bounded by the circle of measurements $T^{m}(\varphi, k)$ ). The boundary conditions are not complete, because the temperature at the radius $R_{m}$ is not known at all times (only known for $t_{\varphi}^{k}=t_{k}+\varphi / \omega$ ). This difficulty is overcome by making an assumption discussed in Section 6.2. Then the solution is extended by continuity toward the surface. Thus, it can be noted that the inverse problem is defined only in $\mathcal{D}_{2}$. Two new sets of conditions are defined:

$$
\text { (Direct part): }\left\{\begin{array}{l}
(r, \varphi, t, k) \in \mathcal{D}_{1} \times\left[t_{k}, t_{k+1}\right] \times \mathbb{N}^{*} \\
T\left(r, \varphi, t_{k}, k\right)=\left\{\begin{array}{ll}
T_{a} & k=1 \\
T\left(r, \varphi, t_{k}, k-1\right) & k \geqslant 2
\end{array} \quad\right. \text { (initial condition) } \\
T\left(R_{m}, \varphi, t_{\varphi}^{k}, k\right)=T^{m}(\varphi, k) \text { (partial boundary conditions) }
\end{array}\right.
$$




$$
\text { (Inverse part): }\left\{\begin{array}{l}
(r, \varphi, t) \in \mathcal{D}_{2} \times\left[t_{k}, t_{k+1}\right] \times \mathbb{N}^{*} \\
T\left(r, \varphi, t_{k}, k\right)=\left\{\begin{array}{ll}
T_{a} & k=1 \\
T\left(r, \varphi, t_{k}, k-1\right) & k \geqslant 2
\end{array}\right. \text { (initial condition) }
\end{array}\right.
$$

\section{Family of solutions}

The space of solution of (1) is an infinite dimensional vector space. Since the problem is linear, a well-known method to find out solutions is to write a linear combination of an infinite family of solutions. In this way, it is demonstrated (proof appended in Appendix A) that the function given by (6) is a solution of (1).

$$
\gamma J_{n}\left(\sqrt{\frac{1}{D \tau}-\frac{i \omega n}{D}} r\right) \exp \left(-\frac{t}{\tau}\right) \exp (\operatorname{in} \varphi)
$$

where $n$ is an integer, $\gamma$ and $\tau$ are complex numbers, and $J_{n}$ is the $n$th Bessel function of the first kind defined for a complex variable. The solution is sought as a linear combination of the family of solution defined by (6), such as:

1. The partial boundary conditions of (4) are verified

2. The initial condition of (4) and (5) are verified

These two conditions are treated separately. The solution is written $T=T_{1}+T_{2}$ where the solution $T_{1}$ verifies the condition 1 but takes not into account the initial condition of (4) and (5) and $T_{2}$ verifies the initial condition 2 (minus the initial temperature field introduced by $T_{1}$ ) and vanishes at the radius $R_{m}$. This decomposition is summarized in Figure 2.

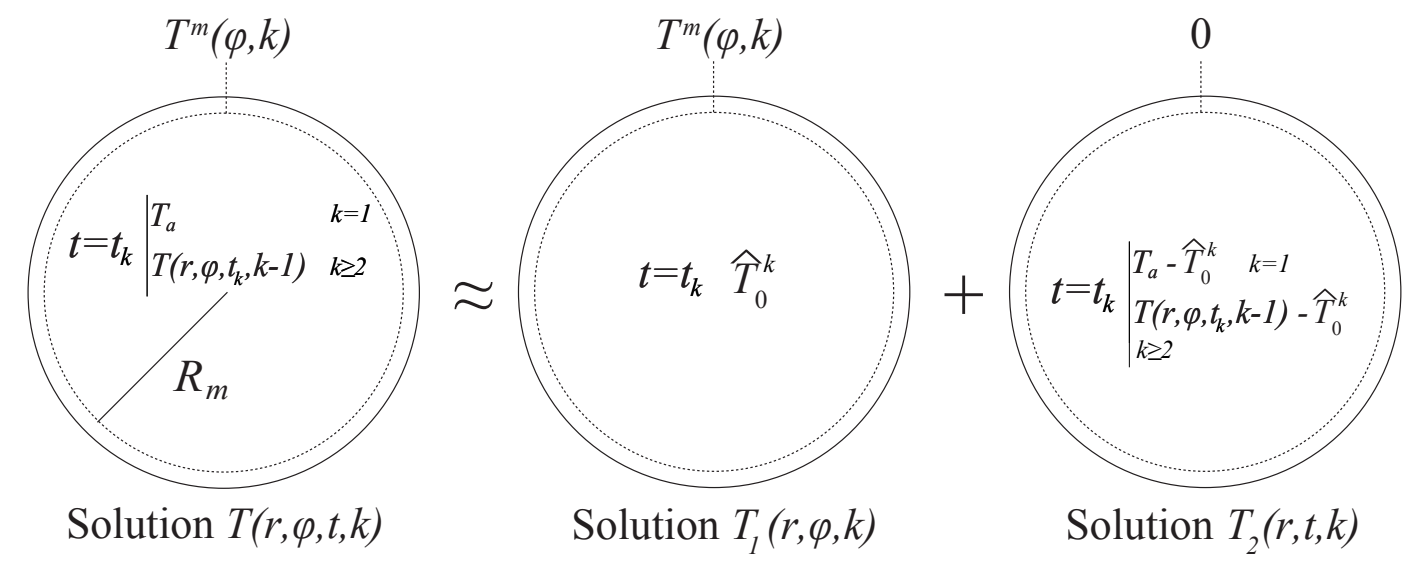

Figure 2: Superposition for each cycle

\section{Approximative analytical solution for each cycle}

An approximative solution of (1) in the form of (6) and verifying approximatively the conditions (4) and (5) is found. For each cycle $k$ :

$$
T(r, \varphi, t, k)=2 \sum_{n=0}^{N_{1}} \operatorname{sinc}^{5}\left(\frac{n}{N_{1}}\right) \operatorname{Re}\left(\widehat{T}_{n}^{k} \frac{J_{n}\left(\zeta_{n} r\right)}{J_{n}\left(\zeta_{n} R_{m}\right)} \exp (i n \varphi)\right)+\sum_{p=1}^{N_{2}} \alpha_{p, k} J_{0}\left(x_{p} \frac{r}{R_{m}}\right) \exp \left(-x_{p}^{2} \frac{D t}{R_{m}^{2}}\right)
$$

where $\operatorname{sinc}(x)=\sin (\pi x) /(\pi x)$, the order of truncation of the sums $N_{1}$ and $N_{2}$ are integers, $\alpha_{p, k}$ are simply obtained by (22), $x_{p}$ are the positive successive zeros of the Bessel function of the order $0\left(J_{0}\left(x_{p}\right)=0\right), \zeta_{n}$ is a complex number such as $\zeta_{n}=(1-i) \sqrt{\omega n / 2 D}$ and $\widehat{T}_{n}^{k}$ are the Fourier coefficients of the measurements done during the cycle $k$. 
Therefore the outputs of the method (the temperature at the outer radius) are given explicitly by :

$$
T^{s}(\varphi, t, k)=2 \sum_{n=0}^{N_{1}} \operatorname{sinc}^{5}\left(\frac{n}{N_{1}}\right) \operatorname{Re}\left(\widehat{T}_{n}^{k} \frac{J_{n}\left(\zeta_{n} R_{s}\right)}{J_{n}\left(\zeta_{n} R_{m}\right)} \exp (i n \varphi)\right)+\sum_{p=1}^{N_{2}} \alpha_{p, k} J_{0}\left(x_{p} \frac{R_{s}}{R_{m}}\right) \exp \left(-x_{p}^{2} \frac{D t}{R_{m}^{2}}\right)
$$

The heat flux at the surface of the roll is given by:

$$
H^{s}(\varphi, t, k)=2 \lambda \sum_{n=0}^{N_{1}} \operatorname{sinc}^{5}\left(\frac{n}{N_{1}}\right) \operatorname{Re}\left(\widehat{T}_{n}^{k} \zeta_{n} \frac{J_{n}^{\prime}\left(\zeta_{n} R_{s}\right)}{J_{n}\left(\zeta_{n} R_{m}\right)} \exp (i n \varphi)\right)+\lambda \sum_{p=1}^{N_{2}} \frac{\alpha_{p, k} x_{p}}{R_{m}} J_{0}^{\prime}\left(x_{p} \frac{R_{s}}{R_{m}}\right) \exp \left(-x_{p}^{2} \frac{D t}{R_{m}^{2}}\right)
$$

The inversion procedure is summarized in Figure 3.

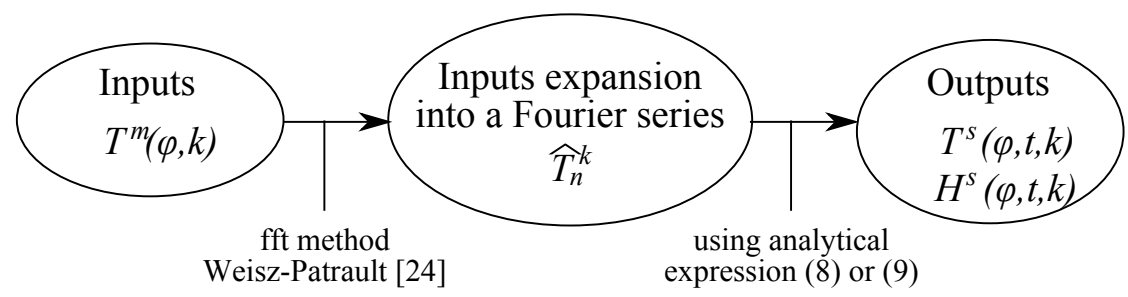

Figure 3: Inversion procedure for each cycle

The solution (7) is by construction a solution of (1), but the sets of conditions (4) and (5) are only approximatively verified. Four assumptions are made:

1. During one cycle the variations of the temperature field are small

2. The initial condition of (4) is only verified under a certain depth and (5) is not verified

3. The temperature field under this depth is almost radial (i.e., independent on $\varphi$ )

4. The initial condition of (4) is only verified for a finite number of points

The result (7) is established in Sections 6 and 8, and these four assumptions are introduced and discussed.

\section{Condition at the radius $\boldsymbol{R}_{m}$}

\subsection{Measurement restrictions}

The solution $T$ is sought in the form $T=T_{1}+T_{2}$. The solution $T_{1}$ only has to match the measurements at the radius $R_{m}$ (i.e., verifying the partial boundary conditions of (4)), and $T_{2}$ only has to correct the solution $T_{1}$ in order to verify the initial condition of (4) and (5). If the temperature on the whole circle was known at any time, it would have been possible to expand the measurements into a Fourier series with time dependent coefficients. The identification of the time dependent Fourier coefficients as sums of time decreasing exponentials would allow to write the solution $T_{1}$ in the form of solutions given by (6). However, the measurement system being a unique local sensor fixed into the body of the roll at the radius $R_{m}$, it provides only the temperatures at different angular positions at successive times. Therefore the time dependent expansion into a Fourier series of the temperature at the radius $R_{m}$ is impossible, because it would need the temperature on the whole circle at any time.

Considering several cycles it is admittedly possible to make a good guess of the temperature at each angular position at any time, with weighting function techniques as proposed by Tseng et al. [25], but the solution would be completely dependent on the choice of the weighting functions. A more simple approach is to work on time scales as proposed in the following.

\subsection{Assumption 1}

In order to determine $T_{1}$ a first assumption is made (because of the restrictions of the measurement system). It is assumed that during one cycle the variations of the temperature field are small. Therefore a natural characteristic time of the problem is the duration of one cycle. Thus the time dependence of $T_{1}$ can be considered for increments of time of one cycle. It means that the temporal evolution of the temperature field during the cycle (the highly 
transient state) cannot be available with such a measurement system. The temporal evolution of the solution $T_{1}$ is only obtained from one cycle to another. However, the slow time dependence of the Eulerian temperature field makes the model widely sufficient for an accurate knowledge intended for industrial use.

Because the solution $T_{1}(r, \varphi, k)$ does not depend on time in the interval $\left[t_{k}, t_{k+1}\right]$, the family of solution can be restricted to:

$$
\gamma J_{n}\left(\sqrt{-\frac{i \omega n}{D}} r\right) \exp (i n \varphi)
$$

$T_{1}(r, \varphi, k)$ is solution of (1) with new boundary conditions (it can be noted that these boundary conditions are complete and therefore well defined):

$$
\left\{\begin{array}{l}
(r, \varphi, k) \in \mathcal{D}_{1} \times \mathbb{N}^{*} \\
T_{1}\left(R_{m}, \varphi, k\right)=T^{m}(\varphi, k) \text { (boundary conditions) }
\end{array}\right.
$$

For each cycle, the measured temperature $T^{m}(\varphi, k)$ is expanded into a Fourier series:

$$
T^{m}(\varphi, k)=\sum_{n=-N_{1}}^{N_{1}} \widehat{T}_{n}^{k} \exp (\operatorname{in} \varphi)
$$

where for each cycle the Fourier coefficient is:

$$
\widehat{T}_{n}^{k}=\frac{1}{2 \pi} \int_{0}^{2 \pi} T^{m}(\varphi, k) \exp (-i n \varphi) d \varphi
$$

The coefficients (13) can be computed from the measurements by using fast fourier transform (fft) as described by Weisz-Patrault et al. [24]. However (especially for the first few cycles), it can be noted that during a cycle the temperature field increases. At the end of the cycle the measured temperature is higher than at the beginning even if the temperature is measured at the same angular position. Therefore the measured temperature $T^{m}(\varphi, k)$ is not exactly $2 \pi$ periodic. The discontinuity between the temperature at the end and at the beginning of each cycle creates a Gibb's effect in the expansion into a Fourier series (large oscillations near the discontinuity). This wellknown effect can be reduced by applying a filter. Lanczos [26] and Acton [27] proposed a filter by multiplying by a gate function in the expansion:

$$
T^{m}(\varphi, k) \simeq \sum_{n=-N_{1}}^{N_{1}} \operatorname{sinc}^{5}\left(\frac{n}{N_{1}}\right) \widehat{T}_{n}^{k} \exp (\operatorname{in} \varphi)
$$

where $\operatorname{sinc}(x)=\sin (\pi x) /(\pi x)$. The remaining oscillations (at the beginning and at the end) are simply removed from the signal. For each cycle, the function (15) is a solution of (1) in the form (6) and which matches the measurements at the radius $R_{m}$ (i.e., verifying boundary conditions (11)).

$$
T_{1}(r, \varphi, k)=\sum_{n=-N_{1}}^{N_{1}} \operatorname{sinc}^{5}\left(\frac{n}{N_{1}}\right) \widehat{T}_{n}^{k} \frac{J_{n}\left(\zeta_{n} r\right)}{J_{n}\left(\zeta_{n} R_{m}\right)} \exp (\operatorname{in} \varphi)
$$

where $N_{1}$ is an integer and $\zeta_{n}=\sqrt{-i \omega n / D}=(1-i) \sqrt{\omega n / 2 D}$. The solution is exactly the solution of the steady heat equation, but updated at each cycle.

\section{Edge effect}

The coefficients $J_{n}\left(\zeta_{n} r\right) / J_{n}\left(\zeta_{n} R_{m}\right)$ can be seen as amplification factors. Thus, the solution (15) can be understood as a filter of the measured temperatures like the interpretation of Blum and Marquardt [11]. In Figure 4, $\left|J_{n}\left(\zeta_{n} r\right) / J_{n}\left(\zeta_{n} R_{m}\right)\right|$ are displayed versus $r$ for some values of $n$. The parameters are settled to very common rolling conditions. The radius of the roll is $R_{s}=254 \mathrm{~mm}$, and the rotation speed is $\omega=4 \pi \mathrm{rad} / \mathrm{s}$. The filter interpretation is clear, the deeper from the surface of the roll the temperature is measured and the lower the frequencies of the signal are. A typical edge effect is demonstrated, which is usually called the skin thickness. 


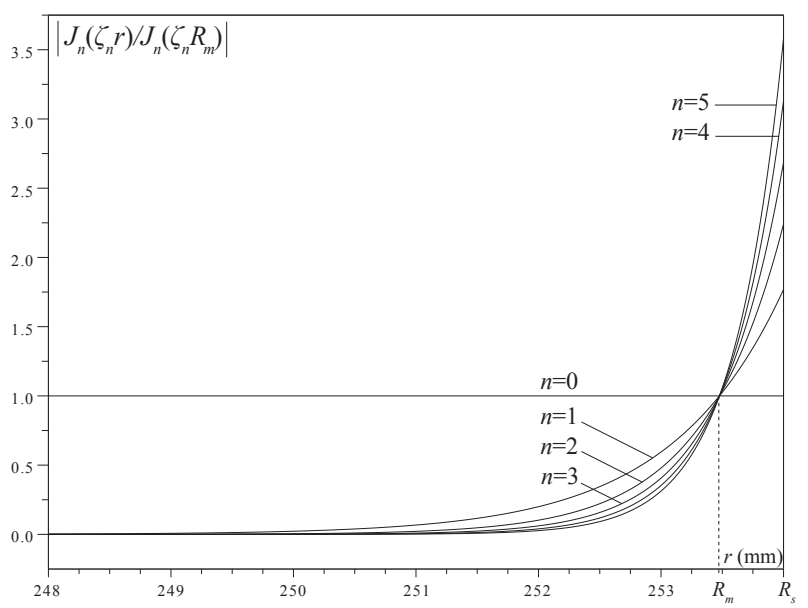

Figure 4: $\left|J_{n}\left(\zeta_{n} r\right) / J_{n}\left(\zeta_{n} R_{m}\right)\right|$ vs $r$ for some values of $n$

The inner radius is chosen in such a way that a signal sufficiently distinct from noise is measured. This choice depends on the rolling conditions. The larger the rotation speed is and the closer from the surface of the roll the local sensor should be. In this very common case, the local sensor is fixed at $0.5 \mathrm{~mm}$. Technologically it is possible to insert a thermocouple at this kind of depth under the surface of the roll.

\section{Initial condition}

\subsection{Assumption 2}

$T_{1}$ does not verify the initial condition (room temperature) of (4) and (5). A corrective solution $T_{2}$ is therefore needed. $T_{2}$ should verify the initial condition minus the initial temperature field introduced by the solution $T_{1}$ and vanish at the radius $R_{m}$. However, the solution $T_{1}$ is not written with a continuous time dependence but for increment of time of one cycle. Therefore the initial temperature field of the solution $T_{1}$ is not defined.

Because of the edge effect demonstrated in Section 7 it is clear that deeper than the skin thickness (for all the duration of each cycle) the temperature field of the solution $T_{1}$ is almost $\widehat{T}_{0}^{k}$. Therefore for each cycle the initial temperature field of the solution $T_{1}$ deeper than the skin thickness is well defined and is $\widehat{T}_{0}^{k}$. Thus the difficulty is overcome by verifying the initial condition of (4) only deeper than the skin thickness. Moreover it should be noted that the condition (5) is not verified, but the influence of initial temperature very close from the surface (in $\mathcal{D}_{2}$ ) is negligible compared with the very large gradients of the solution $T_{1}$ in $\mathcal{D}_{2}$.

\subsection{Assumption 3}

Deeper than the skin thickness the temperature field of the solution $T_{1}$ is almost independent on the angular position $\varphi$ because all the harmonics vanish except the constant one as demonstrated in Figure 4. Therefore the solution $T_{2}$ which verifies the initial condition only deeper than the skin thickness can be sought independent on the angular position $\varphi$. Thus the family of solutions of (1) is restricted to:

$$
\gamma J_{0}\left(\sqrt{\frac{1}{D \tau}} r\right) \exp \left(-\frac{t}{\tau}\right)
$$

Moreover, the solution $T_{2}$ should vanish at the radius $R_{m}$. Therefore, if $x_{p}$ are the successive positive zeros of the Bessel function of the order zero, then:

$$
T_{2}(r, t, k)=\sum_{p=1}^{N_{2}} \alpha_{p, k} J_{0}\left(x_{p} \frac{r}{R_{m}}\right) \exp \left(-x_{p}^{2} \frac{D t}{R_{m}^{2}}\right)
$$

where $N_{2}$ is an integer and $\alpha_{p, k}$ are real numbers. 


\subsection{Assumption 4}

$T_{2}$ is written by considering each cycle as an independent problem. Thus, for the first cycle the initial condition is the room temperature. For the other cycles the initial condition is the temperature field at the end of the previous cycle (which is under the skin thickness $\left.T\left(r, \varphi, t_{k}, k-1\right)=T_{2}\left(r, t_{k}, k-1\right)+\widehat{T}_{0}^{k-1}\right)$. By writing this condition, an expression of $\alpha_{p, k}$ can be found:

$$
\begin{cases}T_{2}\left(r, t_{k}, k\right)=T_{a}-\widehat{T}_{0}^{k} & k=1 \\ T_{2}\left(r, t_{k}, k\right)=T_{2}\left(r, t_{k}, k-1\right)+\widehat{T}_{0}^{k-1}-\widehat{T}_{0}^{k} & k \geqslant 2\end{cases}
$$

In order to solve (18) a final assumption is made. A good approximation is obtained by verifying (18) only for $N_{2}$ radial positions. Thus, a linear equation can be solved. The radial positions are called $u_{q}$ for $q$ varying from 1 to $N_{2}$ taken equally distant from the center to the skin thickness. The expression of $u_{q}$ (used for the numerical results) is appended in Appendix B (Eq.B.1). Therefore by plugging (17) in (18):

$$
\begin{cases}\sum_{p=1}^{N_{2}} \alpha_{p, k} J_{0}\left(\frac{u_{q} x_{p}}{R_{m}}\right)=T_{a}-\widehat{T}_{0}^{k} & k=1 \\ \sum_{p=1}^{N_{2}} \alpha_{p, k} J_{0}\left(\frac{u_{q} x_{p}}{R_{m}}\right)=T_{2}\left(u_{q}, t_{k}, k-1\right)+\widehat{T}_{0}^{k-1}-\widehat{T}_{0}^{k} & k \geqslant 2\end{cases}
$$

The assumptions are summarized in Figure 5.

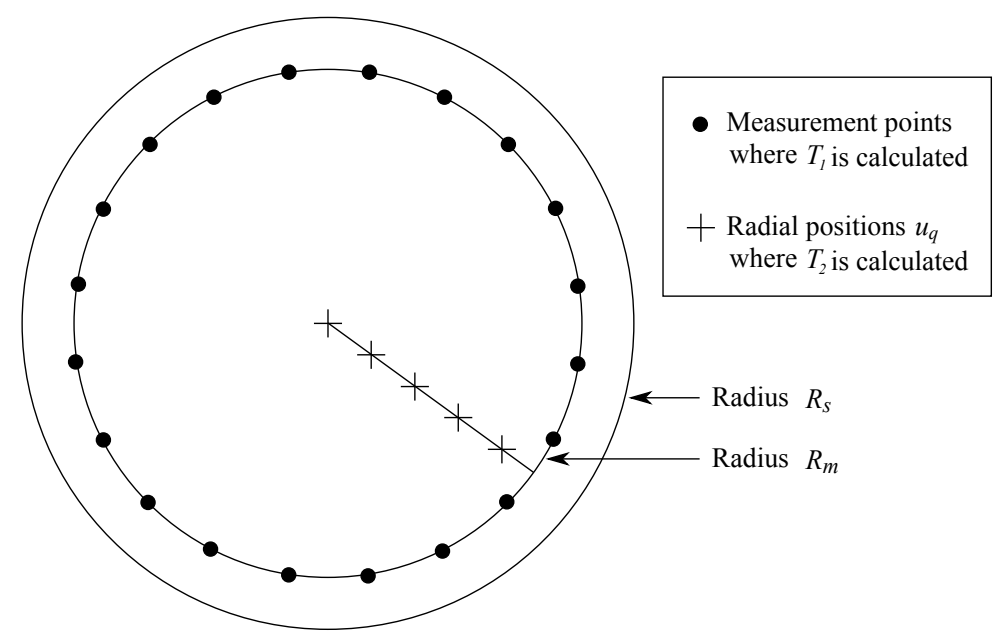

Figure 5: Assumptions

Equation (19) can be written in a matrix form. Let $M$ (size $N_{2} \times N_{2}$ ) be the matrix such as:

$$
M_{q, p}=J_{0}\left(\frac{u_{q} x_{p}}{R_{m}}\right)
$$

and $\alpha_{k}$ denote the vector (size $N_{2}$ ) of $\alpha_{p, k}$ and let $Q_{k}$ and $I\left(\right.$ size $N_{2}$ ) be the vectors such as:

$$
\left\{\begin{array}{l}
I_{q}=1 \\
Q_{q, k}=T_{2}\left(u_{q}, t_{k}, k-1\right)+\widehat{T}_{0}^{k-1}-\widehat{T}_{0}^{k}
\end{array}\right.
$$

The matrix $M$ is invertible and well-conditioned. For instance, for $N_{2}=60$ the determinant is $\operatorname{det}(M)=1.548 \times$ $10^{-4}$ or the condition number is $\operatorname{cond}(M)=25.89$. Thus, the solved matrix form of (19) is:

$$
\begin{cases}\alpha_{k}=\left(T_{a}-\widehat{T}_{0}^{k}\right) \times M^{-1} . I & k=1 \\ \alpha_{k}=M^{-1} \cdot Q_{k} & k \geqslant 2\end{cases}
$$




\section{Commentary}

If a long strip is rolled, it can be considered that the measured temperatures at the radius $R_{m}$ of the current cycle (index $k$ ) are close from the measured temperatures of all the previous cycles. In this case, the assumption 1 (discussed in Section 6.2) is not only true for each cycle taken independently but for all cycles taken together. Therefore, it is not necessary to have for each cycle a new initial condition. In this case $\alpha_{p, k}$ are computed for all cycles with the condition $T_{2}(r, 0, k)=T_{a}-\widehat{T}_{0}^{k}$. Therefore any cycle can be computed without computing all the previous ones. This is used for the simulations presented in Section 10 where the first cycle and the cycle after 10 minutes of rolling are computed.

It is possible to plot for different cycles the function $f_{2}$ given by (23), which multiplied by $T_{a}-\widehat{T}_{0}^{k}$ gives the solution $T_{2}=\left(T_{a}-\widehat{T}_{0}^{k}\right) f_{2}$.

$$
f_{2}(r, t)=\sum_{p=1}^{N_{2}} \beta_{p} J_{0}\left(x_{p} \frac{r}{R_{m}}\right) \exp \left(-x_{p}^{2} \frac{D t}{R_{m}^{2}}\right)
$$

where the vector $\beta=M^{-1}$.I is appended in Appendix B (Table B.5). In Figure 6 the function $f_{2}$ is plotted for successive cycles from 0 to 20000 cycles (or around 2 hours 30 minutes), considering that the rolling parameters are settled to $R_{s}=254 \mathrm{~mm}, R_{m}=253.5 \mathrm{~mm}, \omega=4 \pi \mathrm{rad} / \mathrm{s}$ and $D=13.67 \mathrm{~mm}^{2} / \mathrm{s}$. The influence of the solution $T_{2}$ is negligible when the function $f_{2}$ is near zero. Since the solution $T_{1}$ is exactly the solution of the steady state problem (calculated cycle by cycle) as soon as $T_{2}$ is negligible the solution reaches a steady-state. It appears that the time necessary to get the steady solution is very long for common rolling conditions (around 2h30). The Figure 6 shows however that the importance of the initial condition rapidly vanishes for the skin of the roll $\left(\mathcal{D}_{2}\right)$ whereas it is much slower for the roll core $\left(\mathcal{D}_{1}\right)$.

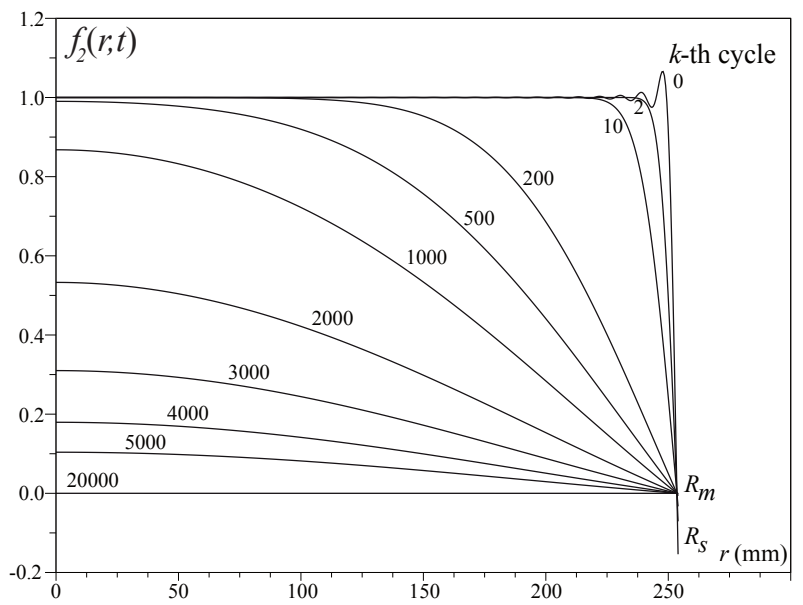

Figure 6: $f_{2}$ vs $r$ from 0 to 20000 cycles (or around 2h30)

\section{Validation of the solution}

\subsection{Direct analytical temperature field}

The accuracy of the present inverse method and its noise sensitivity is demonstrated as follows. A prescribed time dependent temperature field (solution of (1)) is considered and called $T^{p}(r, \varphi, t), p$ meaning prescribed. Therefore at the surface of the roll the temperature is $T^{p}\left(R_{s}, \varphi, t\right)$ and the heat flux is $H^{p}\left(R_{s}, \varphi, t\right)=$ $\lambda\left(\partial T^{p} / \partial r\right)\left(R_{s}, \varphi, t\right)$. Then the temperature at the inner radius $R_{m}$ is extracted from the temperature field. The inputs are the temperature at the radius $R_{m}$ (replacing measurements) considered for successive times (called $\left.t_{\varphi}^{k}=t_{k}+\varphi / \omega\right)$ according to the rotation speed of the roll and the index $k$ of the cycle considered, therefore: $T^{m}(\varphi, k)=T^{p}\left(R_{m}, \varphi, t_{\varphi}^{k}\right)$. The outputs are then calculated and compared to the prescribed temperature (or heat flux) at the surface of the roll. The error $\epsilon$ given in (24) or (25) is used as a percentage to evaluate the quality of the reconstruction.

$$
\epsilon=100 \sqrt{\frac{\int_{0}^{2 \pi}\left[T^{s}\left(\varphi, t_{\varphi}^{k}, k\right)-T^{p}\left(R_{s}, \varphi, t_{\varphi}^{k}\right)\right]^{2} d \varphi}{\int_{0}^{2 \pi}\left[T^{p}\left(R_{s}, \varphi, t_{\varphi}^{k}\right)\right]^{2} d \varphi}}
$$


or if the heat flux is computed:

$$
\epsilon=100 \sqrt{\frac{\int_{0}^{2 \pi}\left[H^{s}\left(\varphi, t_{\varphi}^{k}, k\right)-H^{p}\left(R_{s}, \varphi, t_{\varphi}^{k}\right)\right]^{2} d \varphi}{\int_{0}^{2 \pi}\left[H^{p}\left(R_{s}, \varphi, t_{\varphi}^{k}\right)\right]^{2} d \varphi}}
$$

The prescribed temperature field is:

$$
T^{p}(r, \varphi, t)=2 \operatorname{Re}\left[\sum_{n=0}^{N_{3}} \sum_{j=0}^{N_{4}} a_{n, j} J_{n}\left(\frac{z_{n, j} r}{R_{s}}\right) \exp \left(-\frac{D z_{n, j}^{2} t}{R_{s}^{2}}\right) \exp (\operatorname{in}(\varphi-\omega t))+\sum_{n=0}^{N_{3}} b_{n} J_{n}\left(\zeta_{n} r\right) \exp (\operatorname{in} \varphi)\right]
$$

where $z_{n, j}$ are the successive positive zeros of the functions $h_{n}(x)=\lambda\left(x / R_{s}\right) J_{n}^{\prime}(x)+\operatorname{HTC} \times J_{n}(x)$ and $a_{n, j}$ and $b_{n}$ are complex numbers and $N_{3}$ and $N_{4}$ are integers. The corresponding heat flux is:

$$
H^{p}(r, \varphi, t)=2 \lambda \operatorname{Re}\left[\sum_{n=0}^{N_{3}} \sum_{j=0}^{N_{4}} \frac{a_{n, j} z_{n, j}}{R_{s}} J_{n}^{\prime}\left(\frac{z_{n, j} r}{R_{s}}\right) \exp \left(-\frac{D z_{n, j}^{2} t}{R_{s}^{2}}\right) \exp (\operatorname{in}(\varphi-\omega t))+\sum_{n=0}^{N_{3}} b_{n} \zeta_{n} J_{n}^{\prime}\left(\zeta_{n} r\right) \exp (\operatorname{in} \varphi)\right]
$$

The temperature field $T^{p}(r, \varphi, t)$ given by (26) is in the form of (6) and is therefore an exact solution of the governing equation (1). $a_{n, j}$ and $b_{n}$ can be chosen such as the temperature field $T^{p}(r, \varphi, t)$ corresponds to a roll surrounded by the ambient temperature $T_{a}$ everywhere but in a small angular part $[\pi-\Theta, \pi+\Theta]$ where the surrounding temperature is $T_{\text {ext }}$ (simulating the contact between the strip and the roll). The heat flux entering the roll is defined classically by a heat transfer coefficient HTC. A constant HTC has been considered for simplicity $\left(\mathrm{HTC}=7 \times 10^{-4} \mathrm{~W} \cdot \mathrm{m}^{-2} \cdot \mathrm{K}^{-1}\right)$. This value corresponds to the contact between the roll and the strip (steel/steel) but is physically strongly incorrect outside the contact interval (air/steel or water/steel). Therefore these boundary conditions are very simple compared to real boundary conditions occurring during rolling processes, but for the purpose of validating the present inverse method and its noise sensitivity, they are sufficiently severe boundary conditions because $\Theta, T_{\text {ext }}$ and HTC are adjusted to have a sharp temperature peak quite close from results obtained classically [1] to [7]. Details concerning the calculation of $a_{n, j}$ and $b_{n}$ are appended in Appendix C.

The parameters of the problem are listed in Table 2. The rotation speed is settled to $8 \pi(\mathrm{rad} / \mathrm{s})$ or $6.4 \mathrm{~m} / \mathrm{s}$ considering the radius of the roll, which is the kind of speed commonly used for industrial conditions.

\begin{tabular}{|ll|c|}
\hline \multicolumn{2}{|c|}{ Parameter } & Value \\
\hline$N_{1}$ & $(-)$ & $\begin{array}{c}200 \text { (without noise) } \\
100 \text { (with noise) }\end{array}$ \\
\hline$N_{2}$ & $(-)$ & 60 \\
\hline$N_{3}$ & $(-)$ & 100 \\
\hline$N_{4}$ & $(-)$ & 200 \\
\hline$R_{s}$ & $(\mathrm{~m})$ & 0.254 \\
\hline$R_{m}$ & $(\mathrm{~m})$ & 0.2535 \\
\hline$D$ & $\left(\mathrm{~m}^{2} / \mathrm{s}\right)$ & $6 \times 10^{-6}$ \\
\hline$\lambda$ & $\left(\mathrm{W} \cdot \mathrm{m}^{-1} \cdot \mathrm{K}^{-1}\right)$ & 52 \\
\hline$\omega$ & $(\mathrm{rad} / \mathrm{s})$ & $8 \pi$ \\
\hline$f$ & $(\mathrm{kHz})$ & 10 \\
\hline$T_{a}$ & $(\mathrm{~K})$ & 293.15 \\
\hline$T_{\text {ext }}$ & $(\mathrm{K})$ & 1273.15 \\
\hline $\mathrm{HTC}$ & $\left(\mathrm{W} \cdot \mathrm{m}^{-2} \cdot \mathrm{K}^{-1}\right)$ & $7 \times 10^{4}$ \\
\hline$\Theta$ & $(\mathrm{rad})$ & $\pi / 10$ \\
\hline
\end{tabular}

Table 2: Computing values

\subsection{Reconstruction free from noise}

Using (26), the inputs are produced for three different cycles: the first cycle, the second cycle and the cycle after 10 minutes. The prescribed temperature (and heat flux) at the surface of the roll are given in Figure 7, and the temperature at the inner radius $R_{m}$ (replacing measurements) is given in Figure 8 . The outputs of the method 
compared with the prescribed temperature at the surface of the roll or heat flux entering the roll are given in Figures 9, 10 and 11. The quantified errors are listed in Table 3. When time increases the first assumption of the inverse method exposed in Section 6.2 is more and more verified, therefore the quality of the reconstruction is improved. However even for first cycles the reconstruction is accurate.

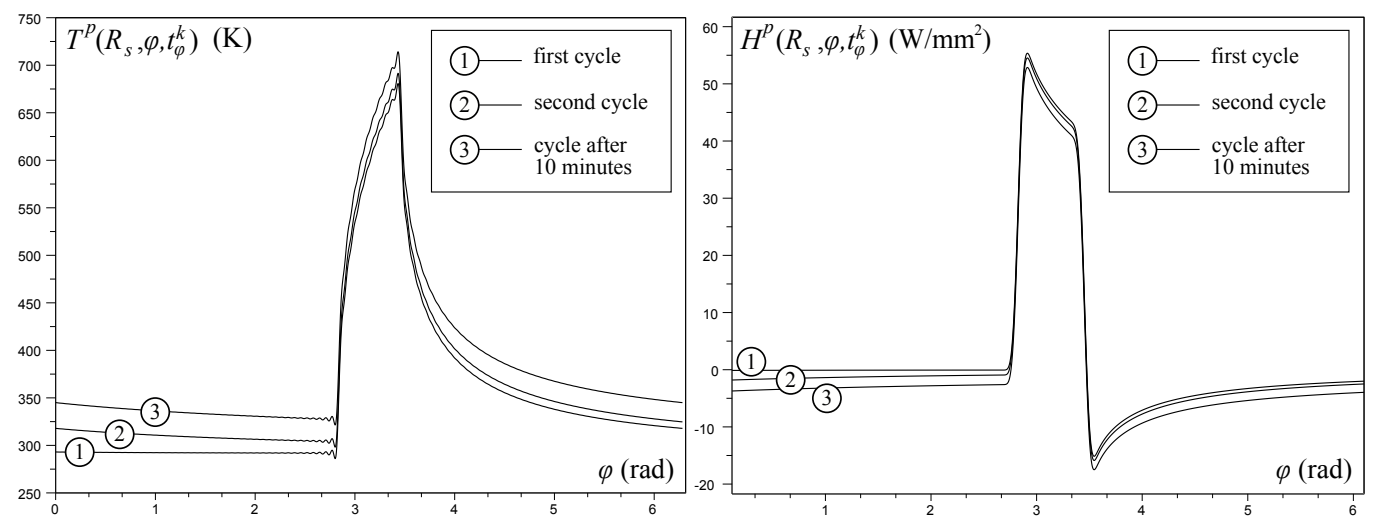

Figure 7: Prescribed temperature and heat flux at radius $R_{S}$ vs $\varphi$

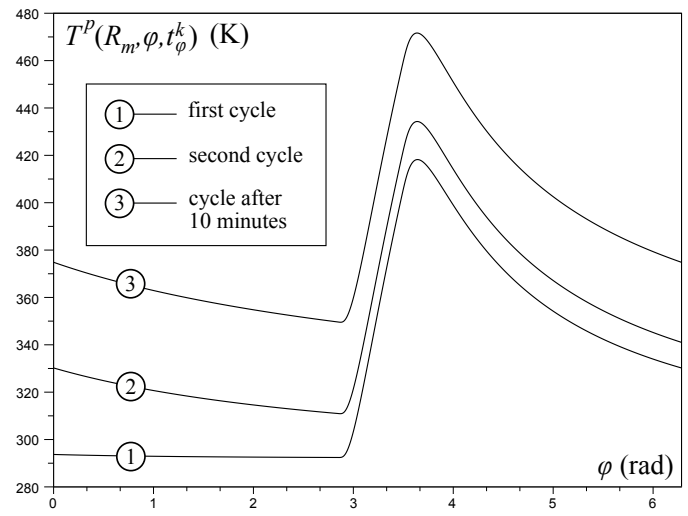

Figure 8: Inputs

\begin{tabular}{|c|l|l|l|l|}
\cline { 2 - 5 } \multicolumn{1}{c|}{} & \multicolumn{3}{c|}{$\epsilon$} \\
\cline { 2 - 5 } \multicolumn{1}{c|}{} & \multicolumn{2}{c|}{ Temperature (Fig.left) } & \multicolumn{2}{c|}{ Heat flux (Fig.right) } \\
\cline { 2 - 5 } \multicolumn{1}{c|}{} & Without noise & With noise & Without noise & With noise \\
\hline First cycle & $3.55 \%$ (Fig.9) & $3.72 \%$ (Fig.12) & $5.82 \%$ (Fig.9) & $8.88 \%$ (Fig.12) \\
\hline Second cycle & $2.19 \%$ (Fig.10) & $2.24 \%$ (Fig.13) & $3.99 \%$ (Fig.10) & $7.77 \%$ (Fig.13) \\
\hline Cycle after 10 minutes & $0.05 \%$ (Fig.11) & $1.16 \%$ (Fig.14) & $0.01 \%$ (Fig.11) & $6.50 \%$ (Fig.14) \\
\hline
\end{tabular}

Table 3: Quantified error 

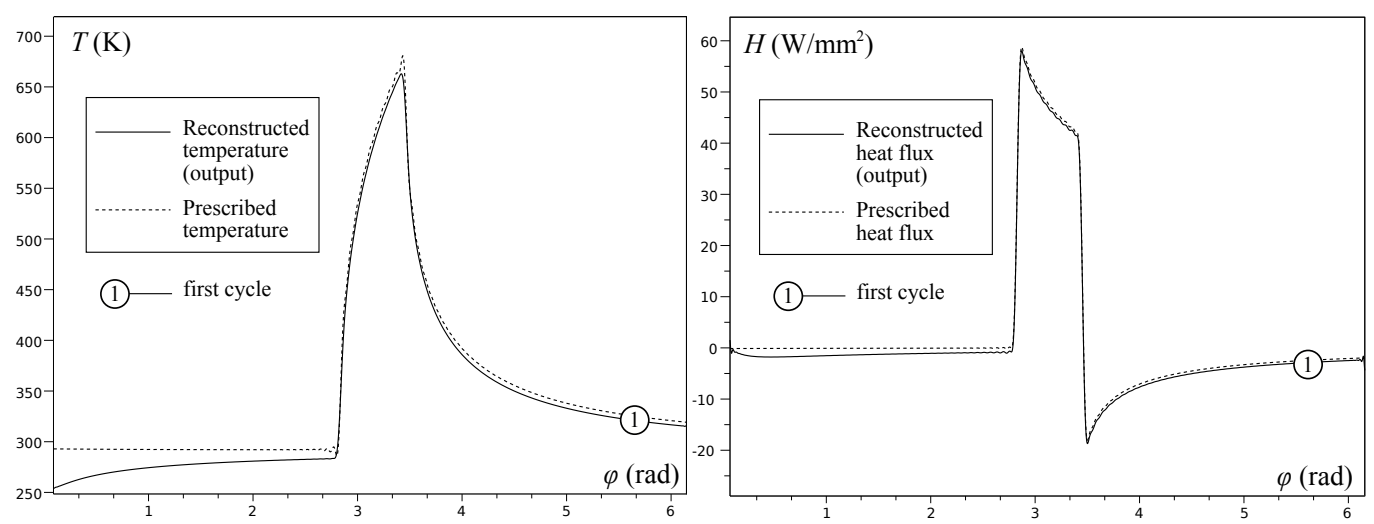

Figure 9: Outputs without noise
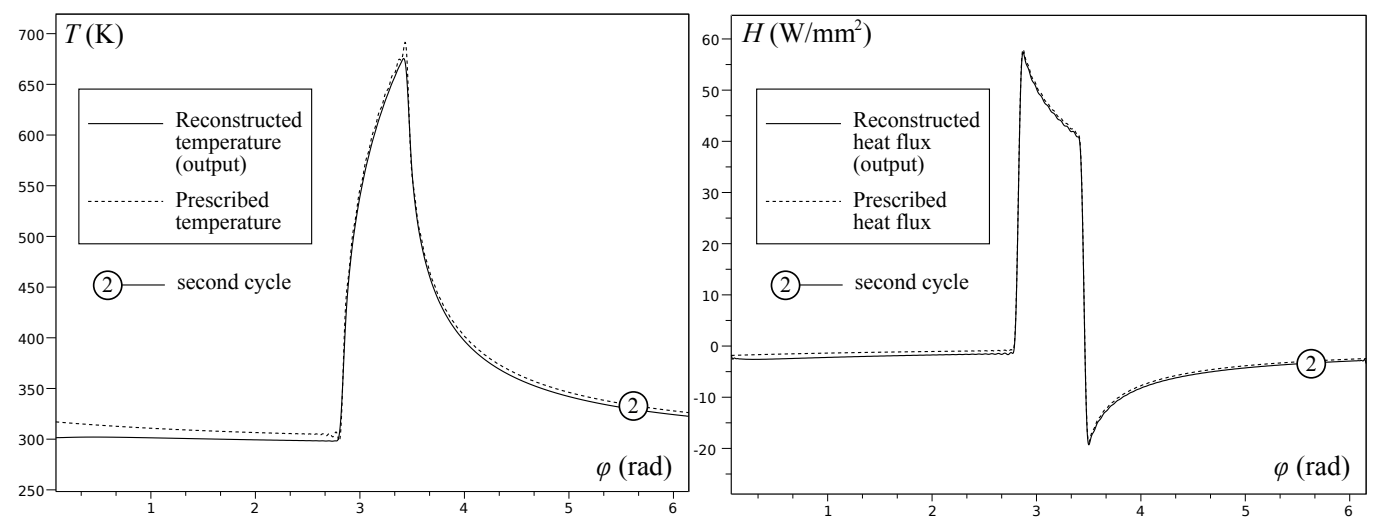

Figure 10: Outputs without noise
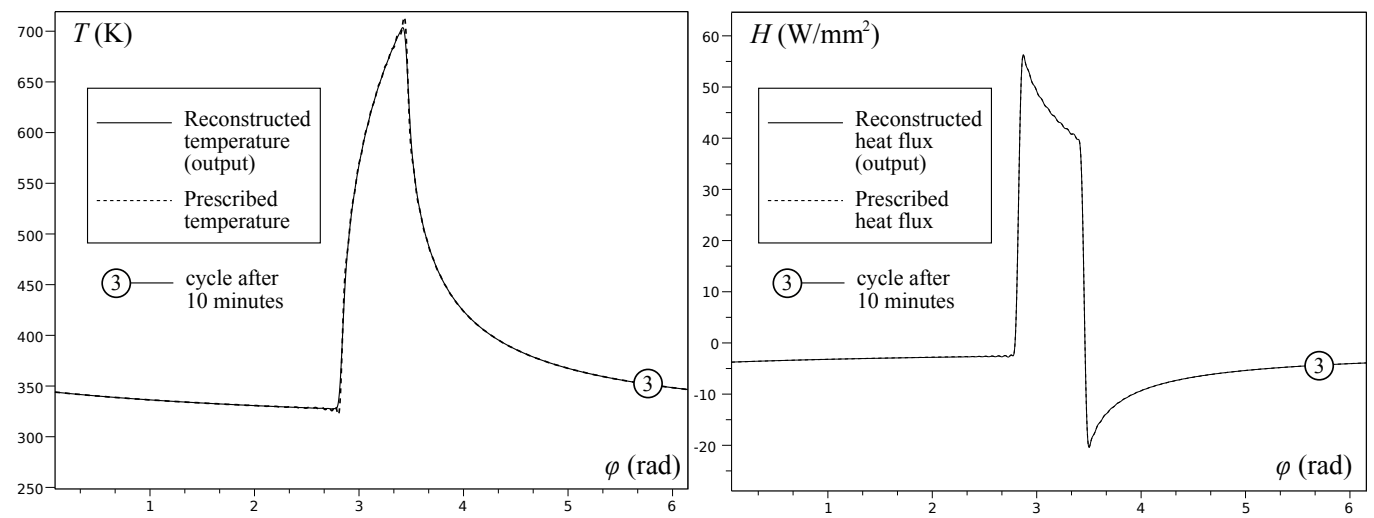

Figure 11: Outputs without noise

\subsection{Noise sensitivity}

The measurements are carried out practically with noise. Artificial noise (uniform law and amplitude $1 \mathrm{~K}$ ) is added to the inputs. Then the reconstruction is calculated like in Section 10.2. However, the order of truncation $N_{1}$ is settled to 100 to avoid a large amplification of noise. No systematic study about the oder of truncation is exposed in this paper. But a basic analysis is presented by Weisz-Patrault et al. [24]. The outputs of the method compared to the prescribed temperature (and heat flux) at the surface of the roll are given in Figures 12, 13 and 
14. The quantified results are listed in Table 3 . The reconstruction is satisfying and therefore the noise sensitivity does not compromise the method. It can be noted that the reconstruction of heat flux is slightly more sensitive than for temperatures, this is due to the derivation.

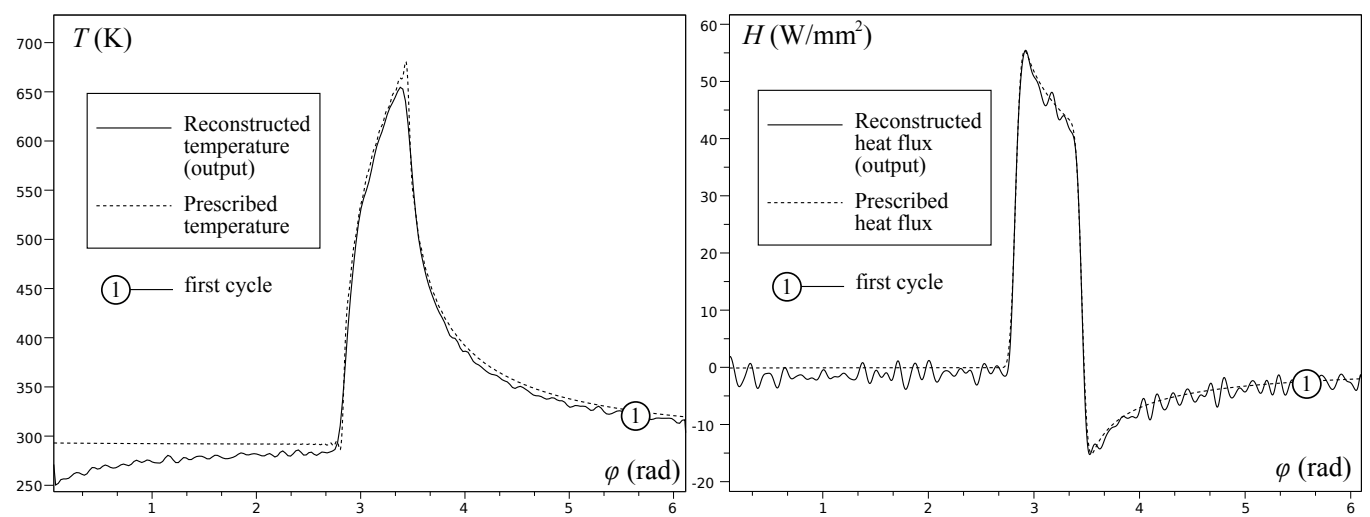

Figure 12: Outputs with noise

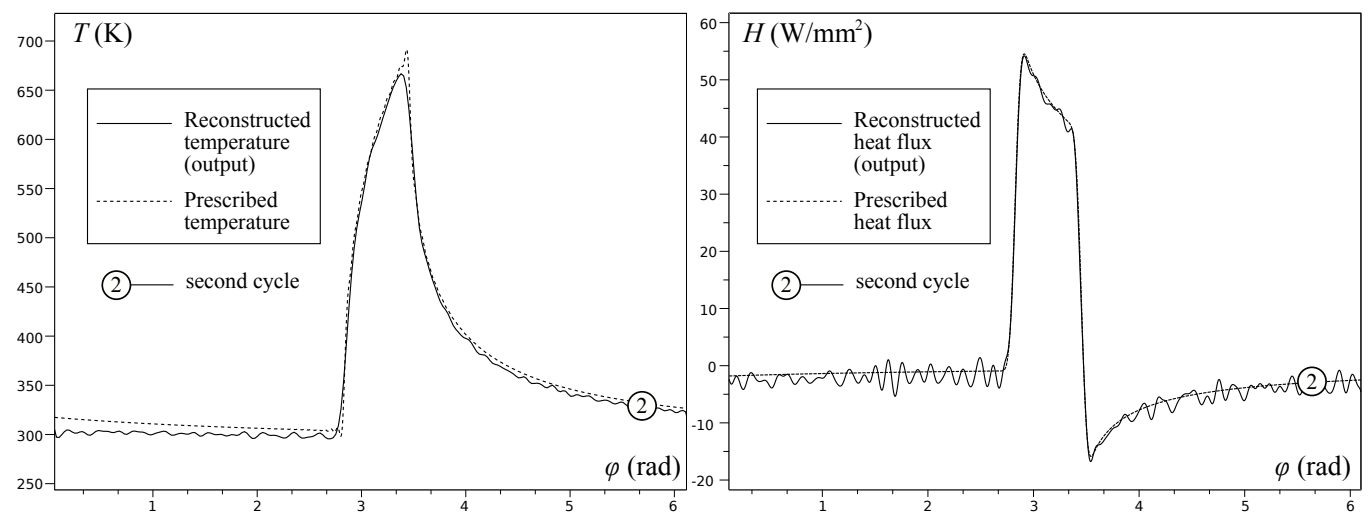

Figure 13: Outputs with noise
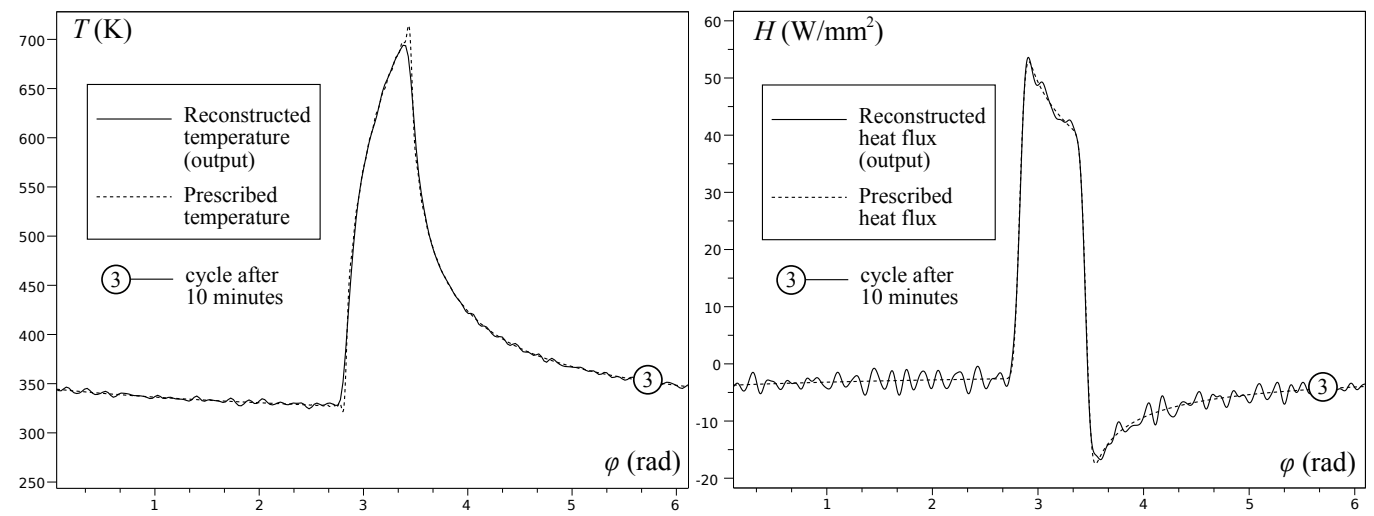

Figure 14: Outputs with noise 


\subsection{Sensor depth}

Technologically the temperature sensor depth is known with an error. Therefore it is a good result if the inverse method is not very sensitive to uncertainties about the sensor depth (considering sharp gradients occurring in rolling processes). Here a $10 \%$ error of the depth is considered. Therefore the error done on $R_{m}$ is $\pm e$, with $e=0.025 \mathrm{~mm}$ considering that the design depth is $0.5 \mathrm{~mm}$. The inputs at different depths are shown in Figure 15. The reconstructed temperature for the first cycle is shown in Figure 16, and the quantified errors are listed in Table.4. The reconstruction of surface temperatures is not very sensitive to a $10 \%$ error of the temperature sensor depth. However, it is slightly more significant for heat fluxes but not critical.

Therefore a calibration process has to considered. A known heat flux can be applied at the surface of the roll. Then the measured temperatures and the computed temperatures inside the roll can be matched by adjusting the sensor depth. The error of sensor depth can be considerably reduced compared with the design value.

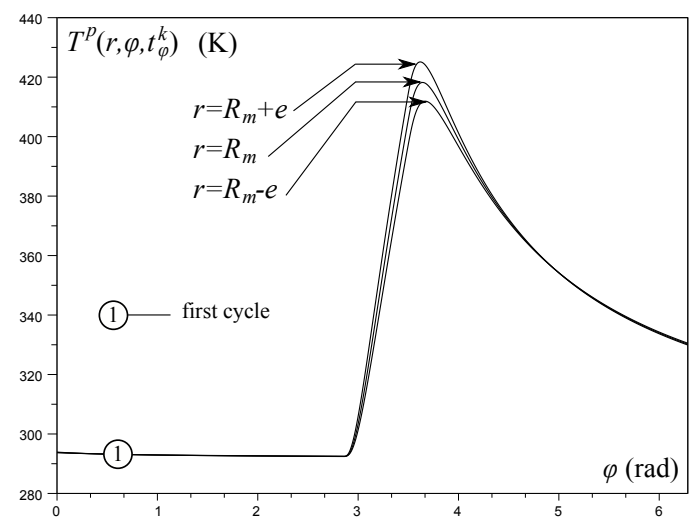

Figure 15: Inputs at different depths
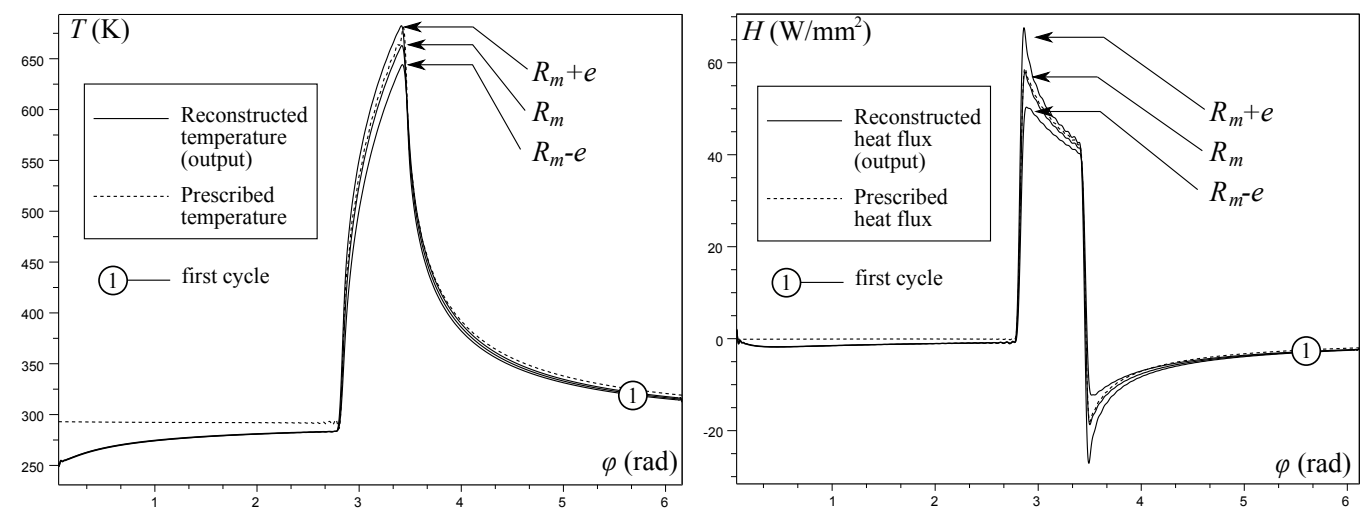

Figure 16: Outputs at different depths

\begin{tabular}{|c|l|l|}
\hline Radius & Temperature & Heat flux \\
\hline$R_{m}$ & $3.5 \%$ & $2.18 \%$ \\
\hline$R_{m}-e$ & $4.3 \%$ & $12.4 \%$ \\
\hline$R_{m}+e$ & $3.9 \%$ & $13.3 \%$ \\
\hline
\end{tabular}

Table 4: Quantified error at different depths (first cycle) 


\section{Computation time}

The computation time is studied to rapidly optimise the industrial parameters during the rolling process. The principle is to write the solution in matrix form. The matrices can be computed off-line (i.e., before the rolling process) and be stocked in a library. The frequency of acquisition is known. Let $N_{\varphi}$ be the number of angular positions $\varphi_{j}\left(j\right.$ varying from 1 to $N_{\varphi}$ ) where the outputs are computed. The solution (8) is written as follows:

$$
T_{k}^{s}=2 \operatorname{Re}\left(A \cdot \widehat{T}^{k}\right)+B \cdot \alpha_{k}+I_{\varphi} \widehat{T}_{0}^{k}
$$

where $T_{k}^{s}$ (size $N_{\varphi}$ ) is the vector of the outputs $T^{s}\left(\varphi_{j}, t_{\varphi_{j}}, k\right), I_{\varphi}$ (size $N_{\varphi}$ ) is a vector of $1, \widehat{T}^{k}$ (size $N_{1}$ ) is the vector of $\widehat{T}_{n}^{k}$, the vector $\alpha_{k}$ (size $N_{2}$ ) is defined in (22) and the matrix $A$ (size $N_{\varphi} \times N_{1}$ ) is:

$$
A_{j, n}=\operatorname{sinc}\left(\frac{n}{N_{1}}\right) \frac{J_{n}\left(\zeta_{n} R_{S}\right)}{J_{n}\left(\zeta_{n} R_{m}\right)} \exp \left(\operatorname{in} \varphi_{j}\right)
$$

and the matrix $B\left(\right.$ size $\left.N_{\varphi} \times N_{2}\right)$ is:

$$
B_{j, p}=J_{0}\left(x_{p} \frac{R_{s}}{R_{m}}\right) \exp \left(-x_{p}^{2} \frac{D}{R_{m}^{2}} t_{\varphi_{j}}^{k}\right)
$$

An effective computation of $B$ is obtained by writing:

$$
B=B 1 . *\left(B 2 .^{\wedge} B 3^{k}\right)
$$

where .^(resp .*) means: power (resp product) term by term. The matrices $B 1, B 2$ and $B 3^{k}\left(\right.$ size $N_{\varphi} \times N_{2}$ ) are:

$$
\left\{\begin{array}{l}
B 1_{j, p}=J_{0}\left(x_{p} \frac{R_{s}}{R_{m}}\right) \\
B 2_{j, p}=\exp \left(-x_{p}^{2} \frac{D}{R_{m}^{2}}\right) \\
B 3_{j, p}^{k}=t_{\varphi_{j}}^{k}=t_{k}+\frac{\varphi_{j}}{\omega}
\end{array}\right.
$$

The matrices $A, B 1$ and $B 2$ are calculated before the acquisition. However $A$ is dependent on the rotation speed, because of $\zeta_{n}$. Therefore several versions of the matrix $A$ (corresponding to several values of $\omega$ ) have to be stocked in a library. The matrix $B 3^{k}$ is clearly dependent on the time $t_{k}$ which is updated at each cycle, however the computation time of this update is negligible because a single value is changed $\left(t_{k}\right)$. Matrices $A$ and $B$ are adapted for the calculation of $H^{s}\left(\varphi, t_{\varphi}, k\right)$.

Therefore the on-line (time consuming) computation is limited to the vectors $\widehat{T}^{k}$ and $\alpha_{k}$ and the scalar $\widehat{T}_{0}^{k}$. Weisz-Patrault et al. [24] used fft in a previous work to compute this kind of integrals $\left(\widehat{T}^{k}\right.$ and $\widehat{T}_{0}^{k}$ ) with a very good precision with a very short computation times. The computation of $\alpha_{k}$ is optimised as follows. Considering (22), the computation of $\alpha_{k}$ requires the computation of $T_{2}\left(u_{q}, t_{k}, k-1\right)$ for $q$ varying from 1 to $N_{2}$. The following matrix $C^{k-1}$ (size $N_{2} \times N_{2}$ ) is computed:

$$
C_{q, p}^{k-1}=J_{0}\left(x_{p} \frac{u_{q}}{R_{m}}\right) \exp \left(-x_{p}^{2} \frac{D}{R_{m}^{2}} t_{k}\right)
$$

An effective computation of the matrix $C^{k-1}$ is obtained with the matrix decomposition:

$$
C^{k-1}=C 1 . *\left(C 2 .^{\wedge} C 3^{k-1}\right)
$$

where the matrices $C 1, C 2$ and $C 3^{k-1}\left(\right.$ size $\left.N_{2} \times N_{2}\right)$ are:

$$
\left\{\begin{array}{l}
C 1_{q, p}=J_{0}\left(x_{p} \frac{u_{q}}{R_{m}}\right) \\
C 2_{q, p}=\exp \left(-x_{p}^{2} \frac{D}{R_{m}^{2}}\right) \\
C 3_{q, p}^{k-1}=t_{k}
\end{array}\right.
$$


The matrices $C 1$ and $C 2$ are computed off-line and the matrix $C 3^{k-1}$ is updated at each cycle by updating $t_{k}$ (computation time negligible). For each cycle the following quantity is then computed in order to update $\alpha_{k-1}$ for the following cycle:

$$
T_{2}\left(u_{q}, t_{k}, k-1\right)=C^{k-1} \cdot \alpha_{k-1}
$$

Using this approach the computation time of the solution (8) is very short. For example the results presented in Section 10 are obtained in around 0.05 second for each cycle with $N_{\varphi}=1000$. The CPU time is obtained for a quadcore $2.8 \mathrm{GHz}$ processor and is the time displayed by Scilab 5.3. Moreover, a program compiled and translated in machine language would significantly reduce the computation time. This kind of computation time could allow a real time close-loop control.

\section{Conclusion}

An inverse analytical method has been developed to estimate the temperature distribution at the surface of the roll and the heat flux entering the roll. The method interprets the measurements of a single thermocouple fully embedded under the surface of the roll. The numerical results presented in this paper are satisfying for an industrial rotation speed. The measurements are carried out practically with noise. Noise sensitivity has been studied by adding artificial random numbers to the inputs, and accuracy has not been compromised. A $10 \%$ error of the temperature sensor depth has also been considered and accuracy has not been compromised either. The main advantage of this contribution, compared to iterative methods, is the very short computation time: 0.05 second for each cycle (the CPU time is obtained for a quadcore $2.8 \mathrm{GHz}$ processor and is the time displayed by Scilab 5.3). Therefore the method is designed for a real time calculation in order to optimise the parameters during industrial rolling processes. An industrial sensor can be developed on the basis of this contribution as a simple tool for experimental studies contributing to the general understanding of thermal behaviour of work rolls during rolling processes.

\section{References}

[1] R. Corral, R. Colas, A. Perez, Modeling the thermal and thermoelastic responses of work rolls used for hot rolling steel strip, Journal of Materials Processing Technology 153 (2004) 886-893.

[2] C.-S. Li, H.-L. Yu, G.-Y. Deng, X.-H. Liu, G.-D. Wang, Numerical simulation of temperature field and thermal stress field of work roll during hot strip rolling, Journal of Iron and Steel Research International 14 (2007) 18-21.

[3] A. Tseng, S. Tong, S. Maslen, J. Mills, Thermal behavior of aluminium rolling, Journal of Heat Transfer 112 (1990) 301-307.

[4] R. Guo, Two-dimensional transient thermal behavior of work rolls in rolling process, Journal of Manufacturing Science and Engineering-Transactions of the ASME 120 (1998) 28-33.

[5] A. Tseng, Finite difference solutions for heat transfer in a roll rotating at high speed, Numerical HeatTransfer 7 (1984) 113-125.

[6] L. Zone-Ching, C. Chang-Cheng, Three-dimensional heat-transfer and thermal-expansion analysis of the work roll during rolling, Journal of Materials Processing Technology 49 (1995) 125-147.

[7] A. Saboonchi, M. Abbaspour, Changing the geometry of water spray on milling work roll and its effect on work roll temperature, Journal of Materials Processing Technology 148 (2004) 35-49.

[8] X. Zhang, Z. Jiang, A. Tieu, X. Liu, G. Wang, Numerical modelling of the thermal deformation of CVC roll in hot strip rolling, Journal of Materials Processing Technology 130 (2002) 219-223.

[9] G. Zhang, H. Xiao, C. Wang, Three-Dimensional Model for Strip Hot Rolling, Journal of Iron and Steel Research, International 13 (2006) 23-26.

[10] P. Montmitonnet, Hot and cold strip rolling processes, Computer methods in applied mechanics and engineering 195 (2006) 6604-6625. 
[11] J. Blum, W. Marquardt, An optimal solution to inverse heat conduction problems based on frequency domain interpretation and observers, Numerical Heat Transfer 32 (1997) 453-478.

[12] M. Raynaud, J. Bransier, A new finite-difference method for the nonlinear inverse heat conduction problem, Numerical Heat Transfer 9 (1986) 27-42.

[13] O. Alifanov, Inverse heat transfer problems, Springer-Verlag, Berlin, pp. 200-203.

[14] J. Beck, B. Blackwell, C. Clair, Inverse heat conduction, ill-posed problems, John Wiley, New York, 1985.

[15] F. Scarpa, G. Milano, Kalman smoothing technique applied to the inverse heat conduction problem, Numerical Heat Transfer 28 (1995) 79-96.

[16] A. Tseng, J. Chang, M. Raudensky, J. Horsky, An Inverse Finite-Element Evaluation of Roll Cooling in Hot-Rolling of Steels, Journal of Materials Processing \& Manufacturing Science 3 (1995) 387-408.

[17] C. Huang, T. Ju, A. Tseng, The estimation of surface thermal-behavior of the working roll in hot-rolling process, International Journal Of Heat And Mass Transfer 38 (1995) 1019-1031.

[18] P. Hsu, Y. Yang, C. Chen, A three-dimensional inverse problem of estimating the surface thermal behavior of the working roll in rolling process, Journal of Manufacturing Science and Engineering-Transactions of the ASME 122 (2000) 76-82.

[19] K. Keanini, Inverse estimation of surface heat flux distributions during high speed rolling using remote thermal measurements, International Journal of Heat and Mass Transfer 41 (1998) 275-285.

[20] W.-L. Chen, Y.-C. Yang, Inverse problem of estimating the heat flux at the roller/workpiece interface during a rolling process, Applied Thermal Engineering 30 (2010) 1247-1254.

[21] R. Tavares, M. Isac, F. Hamel, R. Guthrie, Instantaneous interfacial heat fluxes during the 4 to $8 \mathrm{~m} / \mathrm{min}$ casting of carbon steels in a twin-roll caster, Metallurgical and Materials Transactions B-Process Metallurgy and Materials Processing Science 32 (2001) 55-67.

[22] P. Kotrbacek, M. Raudensky, J. Horsky, M. Pohanka, Experimental study of heat transfer in hot rolling, Revue de Metallurgie-Cahiers d'Informartions Techniques 103 (2006) 333-341. ATS International Steelmaking Conference, Paris, FRANCE, DEC 15-16, 2005.

[23] Y. Kudryavtsev, Unsteady state heat transfer, Iliffe, London, pp. 36-39.

[24] D. Weisz-Patrault, A. Ehralcher, N. Legrand, A new sensor for the evaluation of contact stress by inverse analysis during steel strip rolling, Journal of Materials Processing Technology 211 (2011) 1500-1509.

[25] A. Tseng, F. Lin, A. Gunderia, D. Ni, Roll cooling and its relationship to roll life, Metallurgical Transactions A-Physical Mettalurgy And Materials Science 20 (1989) 2305-2320.

[26] C. Lanczos, Linear differential operators, Van Nostran, Londres and New York.

[27] F. Acton, Numerical methods that (usually) work, The mathematical Association of America, p. 569.

\section{Appendix A. Family of solutions}

The unsteady heat equation relative to an Eulerian reference, expressed in polar coordinates, where the thermal properties of the material are assumed to not depend on the temperature is given by the equation:

$$
\frac{\partial^{2} T}{\partial r^{2}}+\frac{1}{r} \frac{\partial T}{\partial r}+\frac{1}{r^{2}} \frac{\partial^{2} T}{\partial \varphi^{2}}=\frac{1}{D}\left(\frac{\partial T}{\partial t}+\omega \frac{\partial T}{\partial \varphi}\right)
$$

The solution ( $2 \pi$-periodic for $\varphi$ at any time) can be developed in Fourier series:

$$
T(r, \varphi, t)=\sum_{n=-\infty}^{+\infty} F_{n}(r, t) \exp (i n \varphi)
$$


The $n$th Fourier coefficients is sought such as:

$$
F_{n}(r, t)=f_{n}(r) g_{n}(t)
$$

Thus:

$$
g_{n}(t)\left(f_{n}^{\prime \prime}(r)+\frac{1}{r} f_{n}^{\prime}(r)-\frac{n^{2}}{r^{2}} f_{n}(r)\right)=\frac{f_{n}(r)}{D}\left(g_{n}^{\prime}(t)+i \omega n g_{n}(t)\right)
$$

The condition $T(r, \varphi, t)>0$ is verified ( $T$ expressed in Kelvin) therefore:

$$
\left(f_{n}^{\prime \prime}(r)+\frac{1}{r} f_{n}^{\prime}(r)-\frac{n^{2}}{r^{2}} f_{n}(r)\right) \frac{1}{f_{n}(r)}=\frac{1}{D} \frac{1}{g_{n}(t)}\left(g_{n}^{\prime}(t)+i \omega n g_{n}(t)\right)
$$

Both terms are functions of independent variables, thus a complex number $C$ exists such as:

$$
\left\{\begin{array}{l}
f_{n}^{\prime \prime}(r)+\frac{1}{r} f_{n}^{\prime}(r)-\left(C+\frac{n^{2}}{r^{2}}\right) f_{n}(r)=0 \\
g_{n}^{\prime}(t)+(i \omega n-D C) g_{n}(t)=0
\end{array}\right.
$$

Thus:

$$
\left\{\begin{array}{l}
f_{n}(r)=\gamma J_{n}(\sqrt{-C} r) \\
g_{n}(t)=\exp ((D C-i \omega n) t)
\end{array}\right.
$$

where $J_{n}$ is the Bessel function of the first kind of the order $n$ et $\gamma$ a complex number.

By introducing $\tau=-1 /(D C-i \omega n)$ it is obtained that the following function is a solution of (A.1).

$$
\gamma J_{n}\left(\sqrt{\frac{1}{D \tau}-\frac{i \omega n}{D}} r\right) \exp \left(-\frac{t}{\tau}\right) \exp (\operatorname{in} \varphi)
$$

\section{Appendix B. Numerical values}

The radial positions $u_{q}$ are for $q$ varying from 1 to $N_{2}$ :

$$
u_{q}=R_{m} \frac{q-1}{N_{2}}
$$

Thus $u_{q}$ range from 0 to $249.275 \mathrm{~mm}$ which is around the radius of the skin thickness according to the Figure 4 .

\begin{tabular}{|l|c|l|c|l|c|}
\hline$p$ & $\beta_{p}$ & $p$ & $\beta_{p}$ & $p$ & $\beta_{p}$ \\
\hline 1 & 1.6017603 & 11 & 0.4198340 & 21 & 0.2792925 \\
\hline 2 & -1.0640481 & 12 & -0.3994332 & 22 & -0.2697257 \\
\hline 3 & 0.8499229 & 13 & 0.3812070 & 23 & 0.2605739 \\
\hline 4 & -0.7272953 & 14 & -0.3647408 & 24 & -0.2517861 \\
\hline 5 & 0.6451733 & 15 & 0.3497198 & 25 & 0.2433180 \\
\hline 6 & -0.5850795 & 16 & -0.3359009 & 26 & -0.2351319 \\
\hline 7 & 0.5385006 & 17 & 0.3230924 & 27 & 0.2271942 \\
\hline 8 & -0.5009022 & 18 & -0.3111410 & 28 & -0.2194761 \\
\hline 9 & 0.4696192 & 19 & 0.2999224 & 29 & 0.2119517 \\
\hline 10 & -0.4429698 & 20 & -0.2893344 & 30 & -0.2045984 \\
\hline \hline$p$ & $\beta_{p}$ & $p$ & $\beta_{p}$ & $p$ & $\beta_{p}$ \\
\hline 31 & 0.1973955 & 41 & 0.1303318 & 51 & 0.0651469 \\
\hline 32 & -0.1903252 & 42 & -0.1238687 & 52 & -0.0584356 \\
\hline 33 & 0.1833701 & 43 & 0.1174123 & 53 & 0.0516561 \\
\hline 34 & -0.1765158 & 44 & -0.1109563 & 54 & -0.0448147 \\
\hline 35 & 0.1697488 & 45 & 0.1044914 & 55 & 0.0378854
\end{tabular}




\begin{tabular}{|c|c|c|c|c|c|}
\hline 36 & -0.1630565 & 46 & -0.0980124 & 56 & -0.0308898 \\
\hline 37 & 0.1564265 & 47 & 0.0915102 & 57 & 0.0237728 \\
\hline 38 & -0.1498492 & 48 & -0.0849808 & 58 & -0.0166182 \\
\hline 39 & 0.1433137 & 49 & 0.0784137 & 59 & 0.0091932 \\
\hline 40 & -0.1368109 & 50 & -0.0718065 & 60 & -0.0026463 \\
\hline
\end{tabular}

Table B.5: $\beta_{p}$

\section{Appendix C. Direct analytical solution}

An analytical solution is sought for the problem of a roll heated by a surrounding temperature which creates an heat flux equal to the difference between the surrounding temperature and the temperature at the surface of the roll multiplied by a heat transfer coefficient. Here HTC is taken constant which is discussed in Section 10. The surrounding temperature (called $T^{*}$ ) is everywhere $T_{a}$ but in a small angular part $[\pi-\Theta, \pi+\Theta]$ where it is $T_{\text {ext }}$.

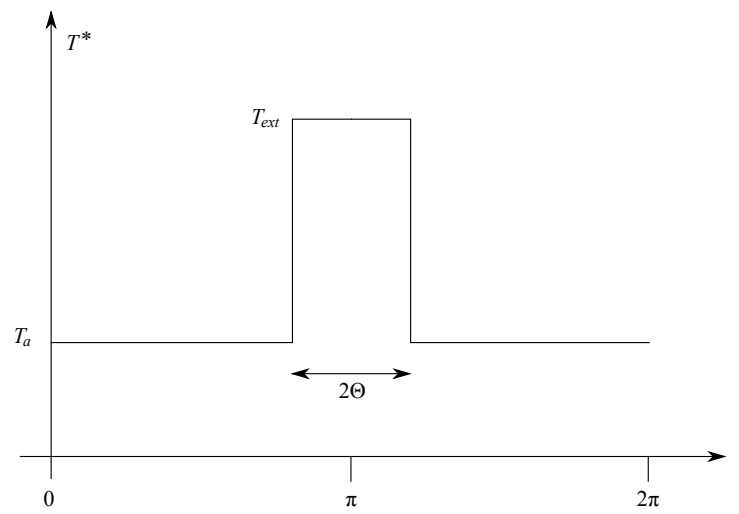

Figure C.17: Surrounding temperature: $T^{*}$

Therefore an expansion into a Fourier series gives:

$$
T^{*}(\varphi)=\sum_{n=-N_{3}}^{N_{3}} \delta_{n} \exp (\operatorname{in} \varphi)
$$

where the order of truncation $N_{3}$ is settled to 100 to avoid long computation times and:

$$
\left\{\begin{array}{l}
\delta_{0}=T_{a}+\frac{\left(T_{e x t}-T_{a}\right) \Theta}{\pi} \\
\delta_{n}=\frac{T_{e x t}-T_{a}}{2 \pi n}[\exp (-i n(\pi-\Theta))-\exp (-i n(\pi+\Theta))]
\end{array}\right.
$$

The solution of the problem is sought in the form of (6):

$$
T^{p}(r, \varphi, t)=\sum_{n=-N_{3}}^{N_{3}} \sum_{j=0}^{N_{4}} a_{n, j} J_{n}\left(\sqrt{\frac{1}{D \tau_{n, j}}-\frac{i \omega n}{D} r}\right) \exp \left(\frac{-t}{\tau_{n, j}}\right) \exp (\operatorname{in} \omega)+\sum_{n=-N}^{N} b_{n} J_{n}\left(\zeta_{n} r\right) \exp (i n \omega)
$$

where $a_{n, j}$ and $b_{n}$ are complex numbers and $N_{4}$ is an integer.

The boundary condition is:

$$
\lambda \frac{\partial T^{p}}{\partial r}\left(R_{s}, \varphi, t\right)=\mathrm{HTC} \times\left(T^{p}\left(R_{s}, \varphi, t\right)-T^{*}\right)
$$

It is convenient to introduce:

$$
z_{n, j}=R_{s} \sqrt{\frac{1}{D \tau_{n, j}}-\frac{i \omega n}{D}}
$$


The boundary condition (C.4) gives:

$$
\frac{\lambda z_{n, j}}{R_{s}} J_{n}^{\prime}\left(z_{n, j}\right)+\mathrm{HTC} \times J_{n}\left(z_{n, j}\right)=0
$$

The $z_{n, j}$ are calculated numerically by taking the successive positive zeros of the functions:

$$
h_{n}(x)=\lambda \frac{x}{R_{s}} J_{n}^{\prime}(x)+\mathrm{HTC} \times J_{n}(x)
$$

Figure C.18 shows an example.

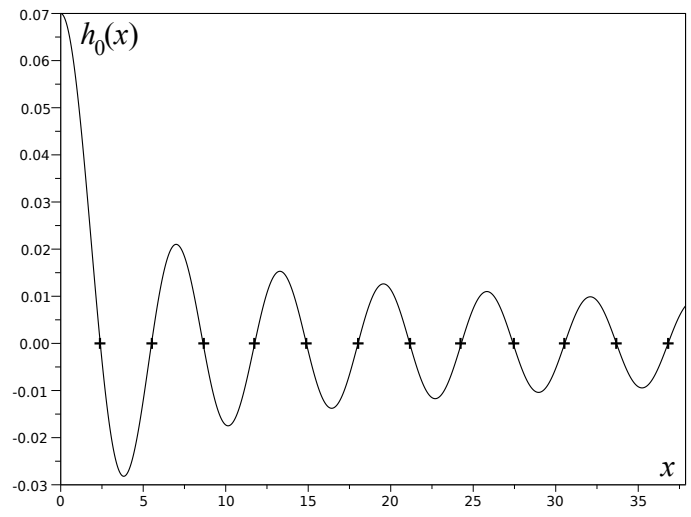

Figure C.18: Successive zeros of $h_{0}(x)$

The boundary condition (C.4) gives also for terms independent on time:

$$
\left\{\begin{array}{l}
b_{0}=\delta_{0} \\
b_{n}=\frac{\mathrm{HTC} \times \delta_{n}}{\lambda \zeta_{n} J_{n}^{\prime}\left(\zeta_{n} R_{s}\right)+\mathrm{HTC} \times J_{n}\left(\zeta_{n} R_{s}\right)}
\end{array}\right.
$$

The initial condition is:

$$
T^{p}(r, \varphi, 0)=T_{a}
$$

The initial condition (C.9) gives:

$$
\left\{\begin{array}{l}
\sum_{j=0}^{N_{4}} a_{0, j} J_{0}\left(\frac{z_{0, j} r}{R_{s}}\right)=T_{a}-b_{0} \\
\sum_{j=0}^{N_{4}} a_{n, j} J_{n}\left(\frac{z_{n, j} r}{R_{s}}\right)=-b_{n} J_{n}\left(\zeta_{n} r\right)
\end{array}\right.
$$

The coefficients $a_{n, j}$ are determined as follows. Eq.(C.10) should be verified for all $r$ from 0 to $R_{s}$. But it is possible to obtain $N_{3}+1$ linear systems to inverse (size $N_{4}+1 \times N_{4}+1$ ) by verifying the equation (C.10) only in $N_{4}+1$ points taken equally distant from 0 to $R_{S}$ included. The initial condition is therefore partially verified. The precision is better and better when $N_{4}$ increase. However to avoid long computation times $N_{4}$ is settled to 200 . 